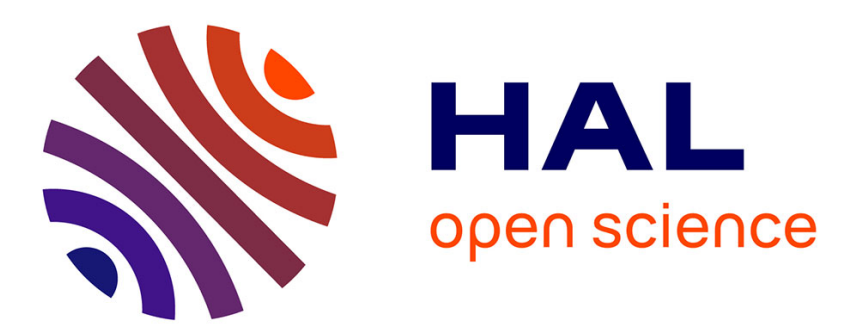

\title{
The Arcuate Fasciculus and language origins: Disentangling existing conceptions that influence evolutionary accounts
}

Yannick Becker, Kep Kee Loh, Olivier Coulon, Adrien Meguerditchian

\section{- To cite this version:}

Yannick Becker, Kep Kee Loh, Olivier Coulon, Adrien Meguerditchian. The Arcuate Fasciculus and language origins: Disentangling existing conceptions that influence evolutionary accounts. 2021. hal$03255234 \mathrm{v} 2$

HAL Id: hal-03255234

https: / hal-amu.archives-ouvertes.fr/hal-03255234v2

Preprint submitted on 29 Oct 2021 (v2), last revised 30 Nov 2021 (v3)

HAL is a multi-disciplinary open access archive for the deposit and dissemination of scientific research documents, whether they are published or not. The documents may come from teaching and research institutions in France or abroad, or from public or private research centers.
L'archive ouverte pluridisciplinaire HAL, est destinée au dépôt et à la diffusion de documents scientifiques de niveau recherche, publiés ou non, émanant des établissements d'enseignement et de recherche français ou étrangers, des laboratoires publics ou privés. 
Keywords: language network, language evolution, frontal terminations, temporal terminations, discontinuity, continuity, lateralization, nomenclature, terminology, hemispheric specialization, comparative anatomy, white matter, tractography, evolutionary neuroscience, monkey, nonhuman primate

Abbreviations: AF (Arcuate Fasciculus), SLF (Superior Longitudinal Fasciculus), MdLF (Middle Longitudinal Fasciculus), dMRI (diffusion magnetic resonance imaging), NHP (Nonhuman primate)

Highlights:

1) Existing conceptions of the AF are disentangled to derive a current model

2) Data indicates a continuity in $A F^{\prime}$ 's frontal and temporal terminations across primates

3) Data indicates a continuity in the AF's strength relative to the Ventral pathway

4) More data is needed to conclude about the (dis)continuity of AF's lateralisation

5) Language evolution theories should incorporate this continuous AF evolution 


\section{Introduction}

The Arcuate Fasciculus (AF), or the arched/curved bundle in latin, is traditionally regarded as one of the major language pathways in the human brain: It arches around the Sylvian or lateral fissure to connect the key speech production region (Broca's area) in the frontal lobe, with the speech comprehension region (Wernicke's area) in the posterior temporal lobe (Geschwind 1970). Congruent with Geschwind's influential model, decades of subsequent research have generally converged on the AF's critical involvement in language and speech functions (e.g., Catani and Mesulam, 2008; Catani and Schotten, 2012; Dick and Tremblay, 2012). Interestingly, the anatomical homologue of this critical white matter pathway for human language exists in the nonhuman primates, where language is absent (e.g., Eichert et al. 2019; Frey et al. 2014; Rilling et al. 2008, 2012). This crucial phylogenetic link provides a valuable avenue to probe the evolutionary origins of human language i.e., by comparing how the AF differs across human and nonhuman primates. Indeed, in the past decade, the emergent field of nonhuman primate or comparative magnetic resonance imaging (MRI) neuroimaging (Friedrich et al. 2021; Milham et al. 2020) have enabled detailed anatomical and functional comparisons of the AF across humans and various nonhuman primate species, such as the macaque monkey and the chimpanzee (e.g., Balezeau et al. 2020; Mars et al., 2021; Rilling et al. 2008, 2012; Rocchi et al. 2021; Wilson et al. 2015). The overarching aim of this review is to synthesize this exciting new body of work that compares the anatomy and function of the AF in humans versus nonhuman primates, to understand how the Arcuate Fasciculus might, or might not, have changed across primate evolution to support the emergence of language in humans.

In the first part of this review, we tackle a major challenge that confronts present-day researchers seeking to investigate the AF: There is currently poor consensus about the anatomical definition of the $A F$ (e.g., where it terminates in the frontal and temporal lobes), and its nomenclature (e.g., whether it is considered part of, or distinct from the Superior Longitudinal Fasciculus (SLF) or Middle Longitudinal Fasciculus (MdLF)). This is primarily driven by the fact that, since its discovery, the methodologies used to study and delineate the AF (e.g., blunt dissections, diffusion MRI tractography, neural tract-tracing) have been constantly evolving, resulting in different descriptions and naming of the AF (e.g., Oliveira et al., 2021; Vavassori et al., 2021). As such, we start the current review with an overview of how the definition and nomenclature of the AF have evolved along with the emergence of different methodologies in the field. This overview, in disentangling the various existing conceptions of the AF, aims to provide a contemporary definition of the fibre bundle that will guide our subsequent review of existing comparative studies of the primate AF.

The second part of this review will discuss four key findings from recent comparative research that shed light on the question about how the AF has changed in humans relative to nonhuman primates: 1) An expansion of the AF's frontal terminations into the inferior frontal cortex (e.g., Schmahmann and Pandya 2006); 2) An expansion of the AF's temporal terminations exceeding the middle temporal cortex (e.g., Eichert et al. 2019); 3) Greater expansion of the Dorsal Language Pathway (which includes the AF), relative to the Ventral Language Pathway (e.g., Rilling et al., 2008); and 4) An absence of lateralisation in the monkey's AF (e.g., Balezeau et al., 2020). For each finding, we will discuss how the different conceptions of the AF have led to the different views on these questions, and subsequently influenced theorisations about the evolution of language. We will conclude that the collective data point towards a continuity in AF anatomy across primates. Last but not least, this review will open a discussion regarding the existing questions and future directions in the field. 


\section{Part I: Disentangling existing conceptions of the Arcuate Fasciculus}

Differing anatomical descriptions and nomenclatures of the Arcuate Fasciculus (AF) fibre bundle have surfaced since its initial discovery by Reil in 1809 (see Dick and Tremblay 2012; ef. Oliveira et al., 2021; Vavassori et al., 2021 for historical reviews of the AF). To a large extent, the various conceptions result from the various emerging methodologies used to investigate the $\mathrm{AF}$, including post-mortem brain dissections, neural tract-tracing, and more recently, diffusion magnetic resonance imaging (dMRI) tractography. Here, we briefly trace the emergence of various conceptions of the $\mathrm{AF}$, relating them to the methods employed to investigate the tract, to finally arrive at a contemporary definition of the AF to guide our subsequent review of the comparative AF literature.

\section{Early $19^{\text {th }}$ to late $20^{\text {th }}$ century: Post-mortem blunt dissections and the synonimity between Arcuate Fasciculus (AF) and Superior Longitudinal Fasciculus (SLF)}

Post-mortem blunt dissections was the first methodology adopted to investigate the Arcuate Fasciculus (AF). The method briefly involves first, the post-mortem extraction and fixation of the brain, followed by the use of a blunt dissection tool to meticulously peel away the grey and white matter progressively to reveal the extents of the various axon bundles. The blunt dissection method was the dominant method employed throughout the $19^{\text {th }}$ century to study human neuroanatomy and had led to many seminal works and discoveries of that era (e.g., Burdach 1826; Dejerine, Dejerine, and Dejerine 1895; 1901; Gratiolet, 1839; Meynert 1885). While this technique enabled the identification of distinct white matter bundles and their individual courses in the brain, they were limited in 1) making distinctions between axon bundles that run in parallel, or that overlap or cross in their courses; and 2) determining the precise axonal origins and terminations of the bundles (Petrides 2014; Yaşargil et al. 2004).

Indeed, it was through blunt dissections that Johann Christen Reil, in 1809, first discovered the system of longitudinal white matter fibres that arched along the Sylvian (or lateral) fissure to interconnect the frontal, parietal and temporal cortices (Figure 1). Using the same method, Friedrich Burdach further characterised and first named these long arching fibres as the "Bogenbündel" in German, which translates to the "arched bundle" or arcuate fasciculus in Latin (Burdach, 1822). For most of the 19th century, two nomenclatures -- "Arcuate Fasciculus (AF)" and "Superior Longitudinal Fasciculus (SLF)" were synonymously used by the most prominent neuroanatomists to describe the longitudinal frontal-parieto-temporal fibre bundle (Dick and Tremblay, 2012). For instance, while Meynert (1885) used the term AF, Wernicke (1874) and Déjèrine $(1895,1901)$ used both AF and SLF to refer to the same longitudinal fibre bundle. The interest for the SLF/AF fibre bundle rose to prominence at the start of the 20th century as Wernicke (1908) identified it as one of two main associative fibre systems for language. Notably, in Geschwind's influential neurological model of language (1970), the term AF, and not the term SLF, was used to depict the fibre system linking the language production centre (inferior frontal cortex) to the language comprehension centre (posterior middle temporal cortex). Since then, the label AF had received an increased recognition and usage to refer to the classical language pathway (more than the term SLF), even though the terms AF and SLF are generally referred to the same tract bundle until the late 20th century (Figure 1). 


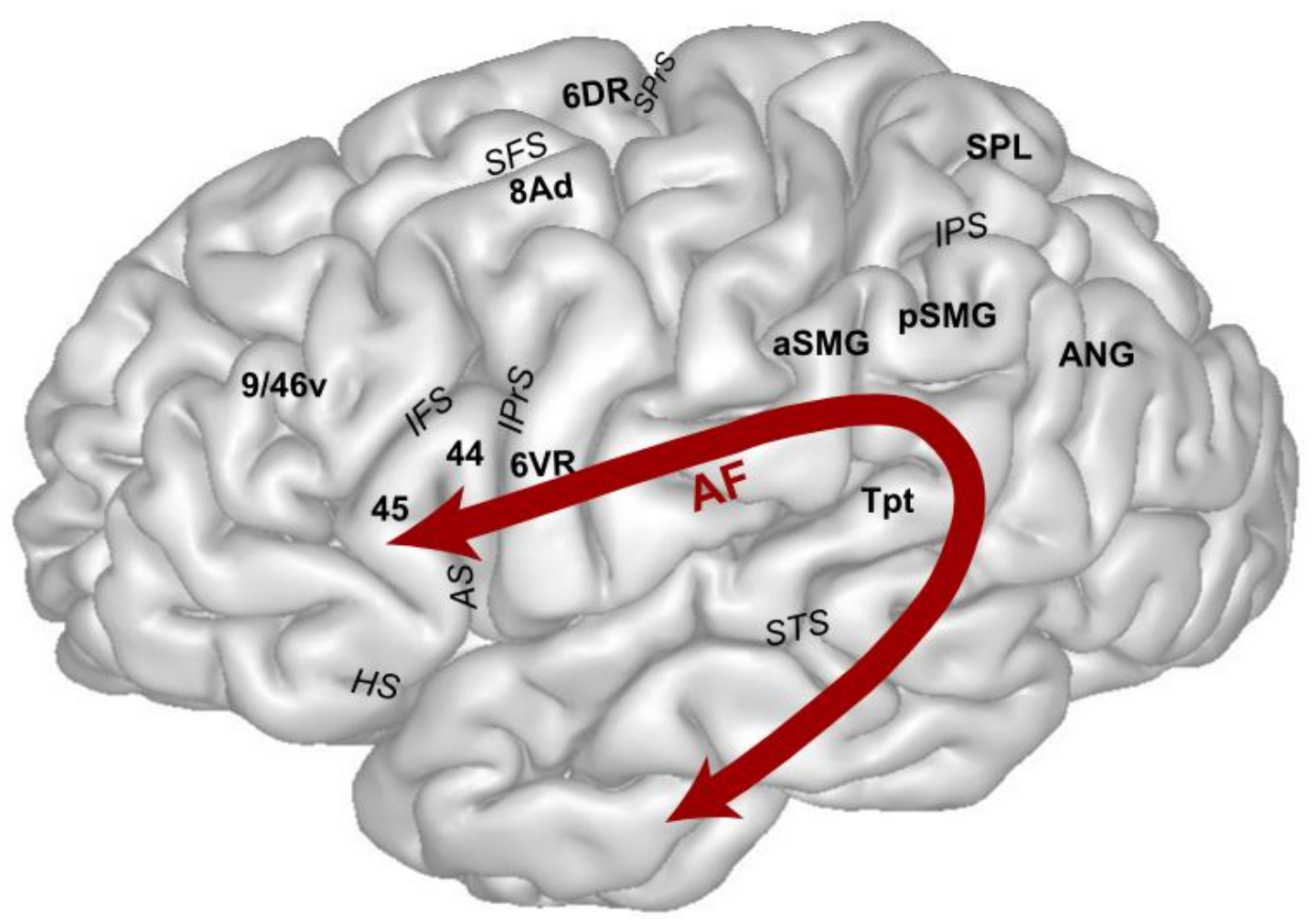

Figure 1: Classic dissection derived AF/SLF Model (e.g., Reil, 1809; Burdach, 1822; Déjèrine, 1895, 1901) in the human brain. AF: Arcuate Fasciculus; SFS=Superior frontal sulcus; SPrS=Superior Precentral Sulcus; IPrS=Inferior Precentral Sulcus; IFS=Inferior frontal sulcus; $A S=$ Ascending branch of SF; HS=Horizontal branch of SF; IPS=Intraparietal Sulcus; Tpt=Temporal-parietal area (BA22); aSMG=anterior supramarginal gyrus (BA40); pSMG=posterior supramarginal gyrus (BA40); $A N G=$ angular gyrus (BA39); $S P L=$ Superior Parietal Lobule (BA7).

From the turn of the 20th century until today, two other methodologies were adopted, alongside blunt dissections, to investigate the Arcuate Fasciculus bundle: neural tract-tracing and diffusion MRI (dMRI) tractography. These techniques have led to dissociate the classic AF/SLF longitudinal fibre bundle into different constituent bundles with distinct courses and terminations. However, due to the differences between the two methods, various models of the AF/SLF fibre system had emerged. Here, we briefly describe each method and its associated models and conceptions of the AF/SLF fibre system.

\section{Late 20th Century: Neural tract-tracing reveals distinctions between the AF and SLF fibre systems}


Neural tract-tracing is a precise, but also highly invasive method for studying brain connections (see Lanciego and Wouterlood, 2020; Saleeba et al., 2019 for recent reviews about the tract-tracing methodology). Briefly, it involves the injection of anatomical tracers into a living brain, which would be transported from the injected region to other regions via interconnecting axons, followed by histological analyses of the brain ex-vivo to visualise the axons and their terminations that have been labelled by the injected tracers. Depending on whether a retrograde or anterograde tracer was administered, the brain region that sends projections into the injected region or the brain region that receives projections from the injected region along with the interconnecting axons, will be labelled, respectively. Hence, using a combination of retrograde and anterograde tracing, the origins and terminations of corticocortical connections associated with a brain region of interest can be precisely determined. As such, contrary to blunt dissection and dMRI tractography, tract-tracing possesses the resolution to distinguish between fibre bundles that overlap or cross in their courses but have different terminations and can therefore also define a monosynaptical connection. Thus, it is commonly regarded as the gold-standard technique for studying brain connections. A key drawback, however, is that tract-tracing is currently only performed in few nonhuman primate subjects due to its invasive nature, which requires the euthanasia of the animal.

Tract-tracing was employed in a series of important studies that resulted in the dissociation of the classical AF/SLF longitudinal fibre system into several distinct fibre bundles based on their origins and terminations in the macaque brain (Frey et al., 2014; Petrides and Pandya, 1984, 1988, 2006, 2009; Schmahmann et al., 2007; Schmahmann and Pandya, 2006; Yeterian et al., 2012 etc.) (Figure 2). In these studies, anatomical tracers were injected into various frontal, parietal and temporal regions in the macaque brain, which have known homologies with the human brain, to study their interconnections. Notably, this body of work has led to a new model of the AF/SLF longitudinal fibre system which consists of four distinct fibre bundles including:

1) the AF, which comprise the fibres that arch dorsally around the Sylvian fissure and connect the posterior temporal cortex with the frontal cortex (the exact frontal and temporal terminations of the AF will be further discussed in Part 2, see also Figure 5); and

2) the SLF I, II and III, which comprise the three longitudinal fibre bundles that interconnect distinct parts of the frontal and parietal cortex and are not arching around the Sylvian fissure (Figure 2).

Since the advent of this approach, the term SLF is used to designate portions of the AF/SLF complex, which are distinct from the AF and which are further distinguished in three different branches I, II and III (Figure 5).

37

38 
Figure 2: Initial Macaque AF and SLF Tract-tracing Model (Petrides and Pandya, 1988,

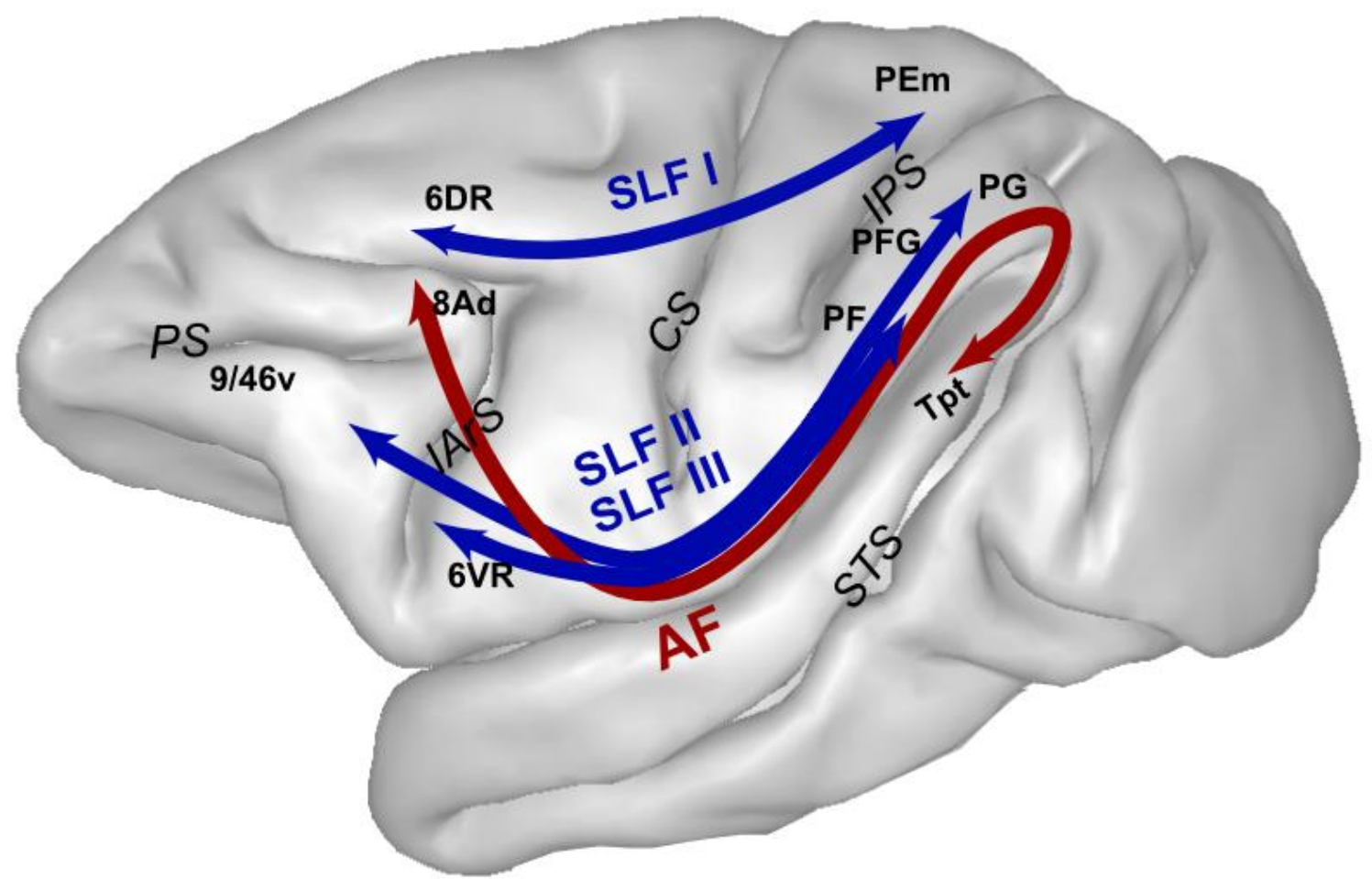

Figure 2: Initial Tract-tracing Model that separated the AF from the SLF in the macaque brain. Adapted from Petrides and Pandya (1988), Schmahmann and Pandya (2006). SLF I, II, III: First, second and third branch of the Superior Longitudinal Fasciculus, AF: Arcuate Fasciculus; IARS=inferior arcuate sulcus, $P S=$ Principal sulcus; $C S$ central sulcus; IPS intraparietal suclus; PF; PEm; PFG; PG are cytoarchitectonic labels for the parietal areas based on Von Economo and Koskinas (1925)

\section{Towards a contemporary conception of the AF: diffusion MRI tractography}

Diffusion MRI (dMRI) tractography is, at present, the only technique available to investigate white matter fibre pathways both in-vivo and ex-vivo, across a large sample of individuals. It involves a form of magnetic resonance imaging that measures the diffusivity of water molecules in the brain. Since water diffusion in the brain is highly constrained by axons (i.e., water tends to flow along the direction of the axons), by tracking the most probable directions of water diffusion voxel-by-voxel, one would be able to reconstruct the main fibre pathways that course between two specific areas in the brain. Alternatively, even the total pathways between all areas in the brain can be reconstructed, yielding a so-called whole-brain tractogram. Note that these reconstructed pathways are essentially "streamlines" that connect vectors of water diffusion computed in each brain voxel and might not necessarily depict true axonal connections. Therefore, tractograms notoriously contain a large number of false positive (Maier-Hein et al. 2017). This is especially so when there are multiple crossing fibres passing 
within a single voxel, which makes it notoriously difficult to determine the actual orientation of fibres (Girard et al. 2020). Additionally, dMRI tractography does not reveal whether a connection is monosynaptic (single axon from origin to termination sites) or polysynaptic (more than one axon interconnecting between origin and termination sites). Due to the limitation to disentangle association pathways like the AF or the SLF (e.g., Girard et al., 2020), most dMRI studies therefore refer to the "Dorsal language pathway or stream" for any pathway connecting frontal to parieto-temporal regions (i.e., AF, SLF II, SLF III) (e.g., Saur et al., 2008). It is opposed to the "Ventral language pathway or stream", curving ventrally around the Sylvian fissure by passing through the extreme capsule and connecting inferior frontal- with temporal areas. This division emulates the well-known visual pathway division (Hickok and Poeppel, 2004). Functionally, the Dorsal pathway is involved in mapping sound to articulation, while the Ventral pathway is involved in mapping sound to meaning (Hickok and Poeppel, 2004; Saur et al., 2008). However recent advances are challenging this dichotomic view and are also highlighting the importance of the dorsal pathway in semantics (Hula et al. 2020). Changes in the AF evolution across primates, concerning the proportional strength between the Dorsal and Ventral pathways, are further discussed in the following section (Question 4, Figure 5). Here we discuss two contemporary and influential conceptions of the classical SLF/AF bundle and how they were derived based on different theoretical underpinnings:

The first conception, which was proposed by Catani and colleagues using dMRI tractography (Catani et al., 2005), is based on priors from classical dissection studies (Figure 3 left panel). This conception names the peri-sylvian longitudinal fibres (both arching and nonarching) collectively as the AF, and divides it into three distinct segments: 1) a long direct segment that connects the posterior part of the superior temporal gyrus (Wernicke's area) to the inferior frontal cortex (Broca's area); 2) an anterior indirect segment that connects Broca's area and inferior parietal cortices; and 3) a posterior indirect segment that links the inferior parietal cortices with the Wernicke's area. This model was obtained via a two-regions-of-interest (ROI) "virtual dissection" approach using diffusion tractography (Catani et al., 2002). First, one ROI was defined that delineated the main body of the AF identified on the fractional anisotropy (FA) image. Next, two other ROIs were defined in the inferior frontal cortex and posterior superior temporal cortex respectively. Fibres were then tracked between each pair of ROIs which yielded the three segments of the AF. Note that the placement of ROIs was strongly influenced by Geschwind's model of the language system (1970) that proposed a pathway, which was termed the AF, linking the Broca's and Wernicke's speech areas in the brain. Indeed, this 3-segment AF model was consistent with neurological models of aphasia and evidence from aphasic patients (e.g., Damasio and Geschwind 1984, Lichtheim, 1885), where the direct long pathway (frontaltemporal) is associated with phonological-related language processes (e.g., rapid word repetitions that do not involve semantic retrieval processes); while the indirect anterior (frontalparietal) and posterior (parietal-temporal) pathways are associated with semantic-related language processes, such as speech comprehension and the production of meaningful words or speech, respectively (Catani et al., 2005). Subsequently, this 3-segment AF model was also validated by other studies using human electrocorticography (Matsumoto et al. 2004), functional connectivity (Holland et al., 2007) and post-mortem dissections (Fernández-Miranda et al., 2008; Lawes et al., 2008).

A second conception comes from researchers who have reconstructed the longitudinal 45 
experiments (Figure 3 right panel) (e.g., Barbeau et al., 2020; Frey et al., 2008; Makris et al., 2005; Warrington et al., 2020). Considering that neural tract-tracing is the gold standard for determining the precise origins and terminations, and axonal courses of brain connections, the macaque model that separates the peri-sylvian longitudinal fibres into the AF, SLF I, II and III bundles (i.e., Frey et al., 2014) would constitute the most anatomically accurate model of the primate longitudinal fibre system at present (Figure 3 right panel). Critically, it remains questionable how generalizable this macaque model is to the human brain, since after all language (which is associated with the AF) is not present in the macaque. One strategy, adopted by Petrides and colleagues (e.g., Frey et al., 2014; Petrides and Pandya, 1988; 2009), was to perform tract-tracing from macaque cortical regions whose human counterparts are known, based on cytoarchitectonics, which facilitates the macaque-human transfer of the anatomical findings. Indeed, by performing in-vivo dMRI tractography in the human brain using carefully defined anatomical landmarks that are known to be homologous to the macaque, recent researchers have been able to replicate the macaque AF, SLF I, II, and III fibre tracts in the human brain (e.g., Barbeau et al., 2020; Frey et al. 2008; Makris et al., 2005).

How then might we reconcile those two legitimate conceptions of the AF? A potential
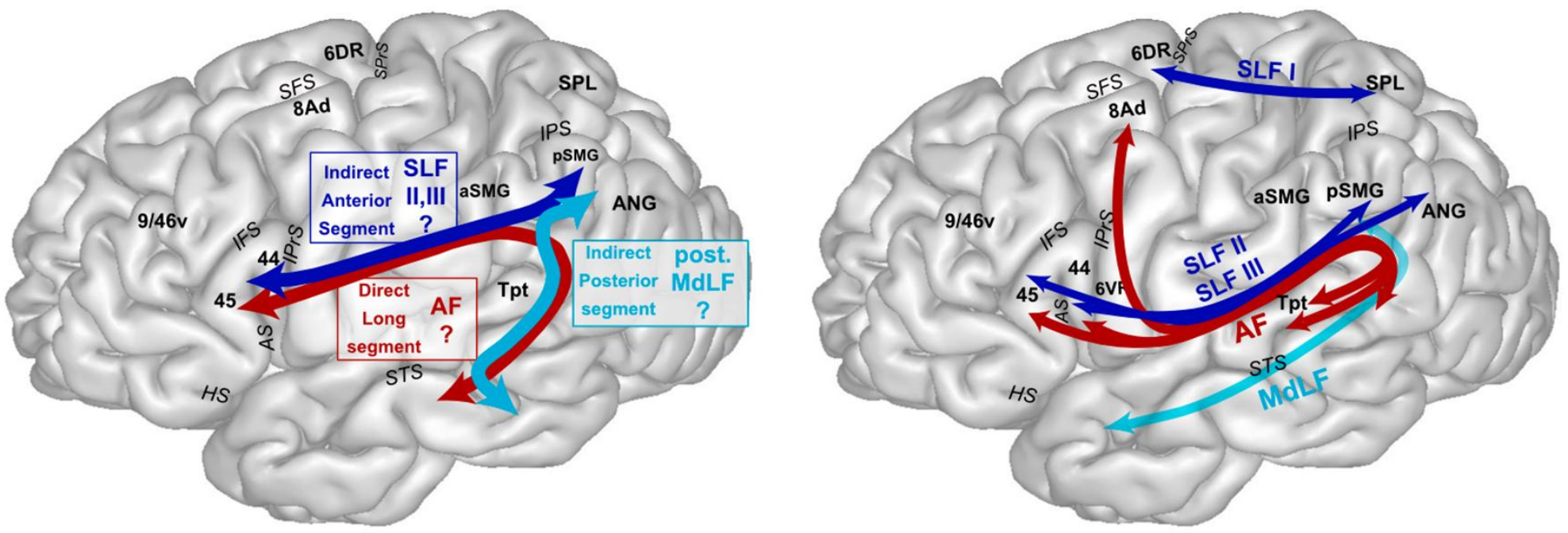

Figure 3: Contemporary Models of the AF fibre pathways in humans. Left: Dissection based human tractography AF/SLF model. Figure adapted from Catani et al., (2005). Right: Tract- 
tracing based human AF/SLF model. Figure adapted from Petrides (2014). Potential correspondences between figures are displayed.

3 SLF I, II, III: First, second and third branch of the Superior Longitudinal Fasciculus, AF: 4 Arcuate Fasciculus, MdLF: posterior segment of the Middle Longitudinal Fasciculus Abbreviation for the human brain. SFS=Superior frontal sulcus; SPrS=Superior Precentral Sulcus; IPrS=Inferior Precentral Sulcus; IFS=Inferior frontal sulcus; AS=Ascending branch of SF; HS=Horizontal branch of SF; IPS=Intraparietal Sulcus; Tpt=Temporal-parietal area (BA22); aSMG=anterior supramarginal gyrus (BA40); $p S M G=$ posterior supramarginal gyrus (BA40); $A N G=$ angular gyrus (BA39); SPL=Superior Parietal Lobule (BA7)

Box 1: Overcoming diffusion MRI tractography limitations

\section{Part II: Four questions about the evolution of the Arcuate Fasciculus across primates}

Current comparative work has revealed four key findings about the unique evolution of the human AF anatomy and compared these to macaques and chimpanzees. First, studies have proposed that the frontal cortical terminations of the AF have changed in the human brain (e.g., Schmahmann and Pandya, 2006). Second, studies have proposed that the temporal cortical terminations of the AF have expanded in the human brain (eg. Eichert et al., 2019). Third, studies have proposed a greater Dorsal (which includes the AF) versus Ventral Pathway expansion across primate evolution (e.g., Rilling et al., 2008). Fourth, studies have proposed an absence of left-lateralisation in the monkeys AF, that is present in humans (e.g., Rocchi et al., 2021). Here, we will re-examine the evidence for these findings with respect to their $\mathrm{AF}$ conceptions and discuss their implications for language evolution theories. 
Figure 4: Illustration of the possible AF's frontal (Question 1) and temporal (Question 2) cortical terminations in the macaque brain

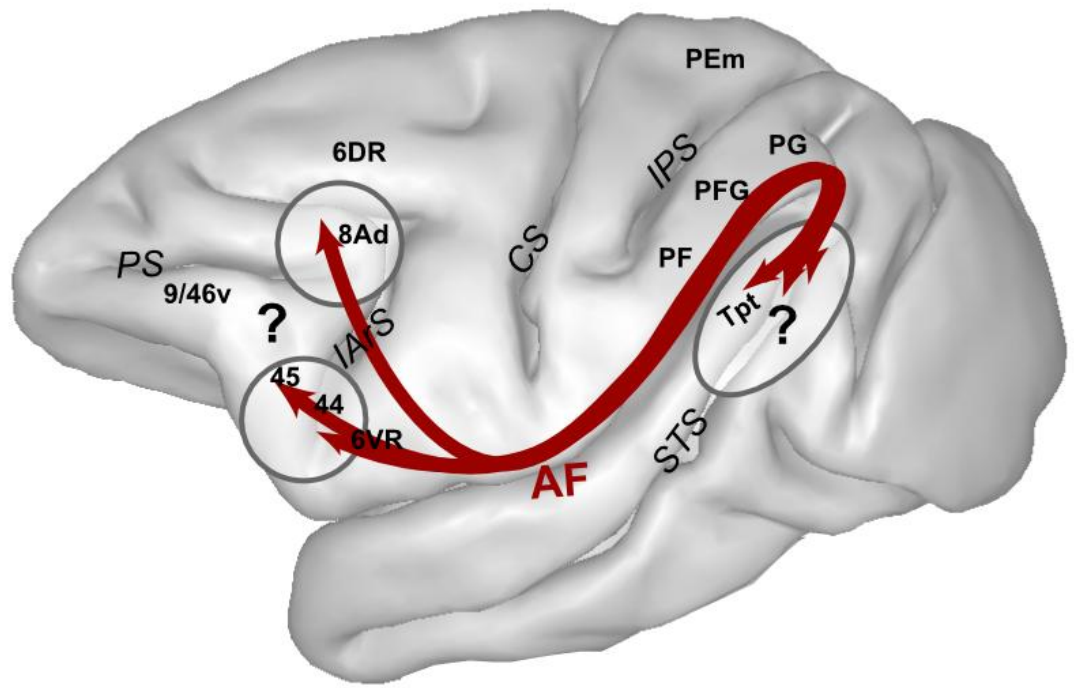

Figure 4: Schematic illustration of the homologue's main language pathways in the macaque monkey brain. Illustration of the course and terminations (arrowheads) of the Arcuate Fasciculus (AF) in red. Grey circles indicate the possible terminations as discussed in the literature (see table 1). Question marks highlight the research questions.

\section{Question 1: Are AF terminations in the inferior frontal cortex (Broca's area) present in the human-, but not in the nonhuman primate brain?}

Classically, the human AF connects to the classical Broca's area in the inferior frontal cortex (Brodmann's areas 44 and 45). These frontal regions are significant for language because they are involved in the high-level control of orofacial and vocal motor responses that are needed for language production (Loh et al. 2020). Direct AF connections to these frontal regions thus provide access to the key motor actions (e.g., articulation, facial expressions, gestures) needed for language expression in humans (Petrides and Pandya, 2009). Whether the same AF frontal projections exist in nonhuman primates (NHPs) is therefore a crucial question that would shed light on why language exists only in humans. Currently, there are several theoretical accounts based on how a lack of AF frontal terminations in the nonhuman primate brain could explain the uniqueness of human language (e.g., Aboitiz and García, 1997; Friederici 2017; Pulvermueller 2018; Schmahmann and Pandya, 2006). However, the most recent data from tract-tracing experiments (e.g., Frey et al., 2014) appear to contradict these accounts as AF connections to macaque areas 44 and 45 were demonstrated. Here, we carefully review past and present tracttracing studies of the macaque peri-sylvian longitudinal fibre system to resolve the question about whether $\mathrm{AF}$ inferior frontal terminations exist in the macaque brain.

Based on the landmark tract-tracing investigation in the macaque brain, Petrides and Pandya (1988) had traced AF terminations into the dorsal part of the frontal cortex (dorsal areas 6, 8A and prefrontal area 46), but not to the inferior frontal cortex, where Broca's area's homologue lies. Instead, the inferior frontal cortex only connected to the parietal cortex via SLF 
II and III. This result remained important in the field over the next two decades: it was included and reproduced in the highly influential atlas of fibre pathways of the macaque brain by Schmahmann and Pandya (2006; see Fig 13-5 on pp 407 for a depiction of the AF) and served as the anatomical reference for several dMRI studies (e.g., Thiebaut de Schotten et al. 2012) (Figure 5 Left panel). Critically, this discovery had led to several theoretical accounts suggesting that the change in AF's frontal projections from dorsal part of the frontal cortex in nonhuman primates to Broca's area in humans could explain the emergence of human language (e.g., Aboitiz and García, 1997; Friederici 2017; Pulvermueller 2018; Schmahmann and Pandya 2006). For instance, Schmahmann and Pandya (2006) proposed that the macaque AF might not be a "language bundle" (pp 408) as it does not connect the homologues of Broca's (inferior frontal cortex) and Wernicke's areas (posterior superior temporal gyrus), but instead the caudal temporal area Tpt (which is associated with auditory-spatial processing) and the dorsal frontal areas (that is associated with oculomotor and attentional control). Due to the lack of a direct dorsal connection of Broca's homologue with the temporal lobe, but rather with the parietal lobe, Aboitiz \& García (1997) proposed the parietal lobe to play a fundamental element in language evolution, linking indirectly the two key structures (Broca's and Wernicke's) by means of the SLF II and III. In their view, it "permitted the development of an interphase between the auditory processing device and a working memory circuit for complex vocalisations that includes inferoparietal and ventral premotor regions". Other theoretical accounts suggested that the absence or reduced degree of the AF's terminations in the inferior frontal cortex (area 44) in NHPs led to the lack of syntax (Friederici, 2017) or action-perception representations (Pulvermueller, 2018) that are necessary for language.

A conceptual change in the views of the AF's frontal terminations happened when a the cytoarchitectonical equivalence of Broca's area was established in the macaque (Petrides et al., 2005), which prompted a series of tract-tracing experiments to reinvestigate the frontal-parietaltemporal pathways with new and more precise tracer injection sites (Petrides, 2014) (Figure 5). Following a detailed comparative analysis of the cytoarchitecture of macaque and human frontal cortex, Petrides and Pandya (Petrides et al. 2005a; b; Petrides and Pandya 1999; 2002) established critical correspondences between the various frontal areas between the two species. In particular, this work revealed for the first time the precise organisation of the homologues of Broca's area 44 and 45 in the macaque brain. With these knowledge, the longitudinal fibre connections between the frontal, parietal and temporal lobe were re-investigated, leading to the major discovery of the existence of long arching fibres that directly connect macaque areas 44 and 45 to the caudal temporal cortex (Frey et al., 2014; Petrides and Pandya, 2009). Petrides explains in his 2014 book, that no terminations to area 44 and 45 were previously found, because the initial injection site was not placed ventrally enough within the temporal lobe (Petrides \& Pandya, 2009; Petrides, 2014) (see Figure 5 right panel that shows each different injection site with an arrowhead and the resulting course with a different colour)

In fact, this work revealed three AF branches projecting to the frontal cortex (see Petrides 2014, pp 160, Fig 52): 1) A dorsal branch that connects from the dorsal part of the superior temporal gyrus to dorsal frontal area $8 \mathrm{Ad}$ and area $6 ; 2$ ) A second branch that connects from the ventral part of the superior temporal gyrus, and the upper bank of the superior temporal sulcus to area 44; and 3) A last branch that connects from the caudal superior temporal gyrus and the adjacent bank of the superior temporal sulcus to area 45 (Figure 5). This result was recently reproduced in comparative diffusion MRI studies, in-vivo and ex-vivo, in macaques and 
chimpanzees (Balezeau et al. 2020; Barrett et al. 2020; Bryant et al. 2020; Eichert et al. 2019, 2020; Rilling et al. 2008; Roumazeilles et al. 2020) and on a functional level using effective connectivity (Rocchi et al., 2021).

As such, based on the above recent tract-tracing data, it is clear that AF connections to the inferior frontal cortex exist in the macaque brain, as in humans, suggesting that the so-called human language pathway between Broca's and Wernicke's areas might be more preserved in nonhuman primates than previously thought. Further supporting this conserved primate AFinferior frontal cortical connectivity, recent studies have also reported common roles of macaque and human frontal areas 44 and 45 in cognitive vocal control (Loh et al., 2017; Loh et al., 2020). As such, contemporary theories that explain the uniqueness of human language based on the absence of inferior frontal projections of the AF must be revised accordingly. If AF frontal terminations are highly conserved, the question arises why language has evolved in humans alone? This important open question remains to be addressed by future research.

Figure 5: Knowledge progress about the macaque Arcuate Fasciculus from tract-tracing experiments.

19

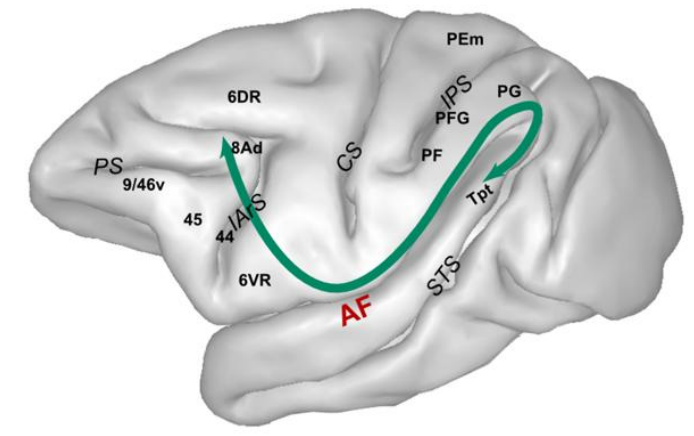

Petrides and Pandya, 1988

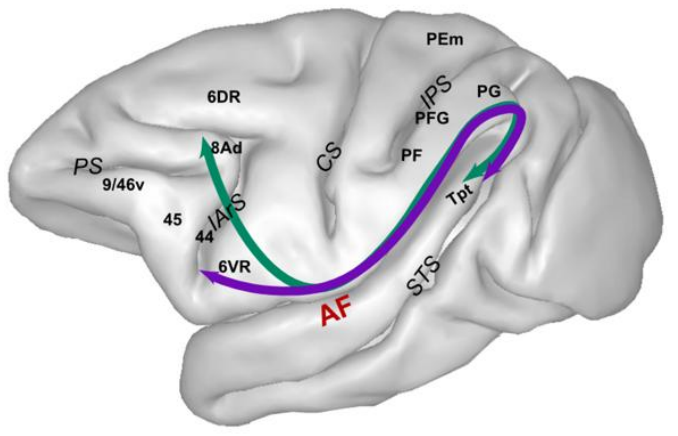

Petrides and Pandya, 2009

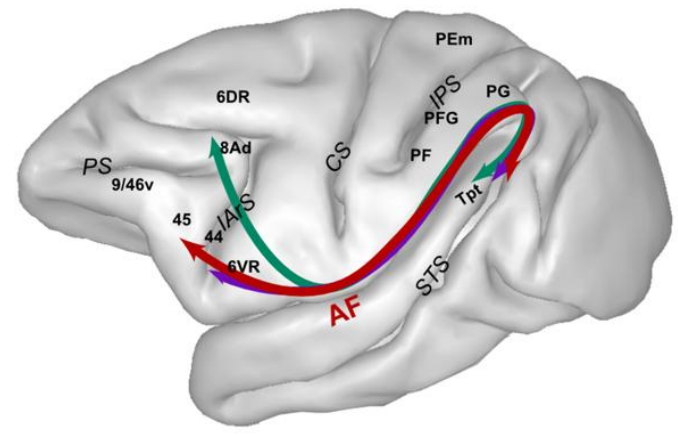

Frey et al., 2014 Schmahmann and Pandya, 2006

Figure 5. Knowledge progress about the macaque Arcuate Fasciculus courses and terminations from in tract-tracing experiments. (Left) Injections into the macaque caudal superior temporal gyrus (area Tpt) reveal the dorsal branch of the AF (Petrides and Pandya, 1988; Schmahmann and Pandya, 2006). (Middle) Injections into the ventral part of Tpt, and the upper bank of the STS reveals a second branch of the AF to area 44 (Petrides and Pandya, 2009; Case 5). (Right) More ventral injections into the caudal superior temporal gyrus and STS reveals a third branch of the AF to area 45 (Frey et al., 2014). Abbreviations: IARS=inferior arcuate sulcus, $P S=$ Principal sulcus; CS central sulcus; IPS intraparietal suclus; PF; PEm; PFG; PG are cytoarchitectonic labels for the parietal areas based on Von Economo and Koskinas (1925).

\section{Question 2: Do human AF terminations extend further into the temporal lobe than in nonhuman primates?}


In the human brain, the AF arches around the Sylvian fissure and extends beyond the posterior part of the temporal cortex, where the Planum Temporale and tpt lies, and beyond the middle temporal gyrus (MTG) into the inferior and anterior temporal cortex (e.g., Catani et al., 2005) (e.g., Figure 3 left panel). This part of the temporal cortex is associated with semantic verbal (or multimodal) information (Binder et al., 2009), and as such, this implies that the human AF provides the frontal speech areas access to semantic information in the MTG. The difference in temporal terminations between human and NHP is therefore crucial, in order to investigate potential evolutionary divergences, which, in turn, influence functional theories about language evolution. It is widely agreed that the human $\mathrm{AF}$ is unique in extending into the middle part of the temporal cortex (e.g., Rilling et al., 2008), even though recent investigations in monkeys also highlight slightly more anterior temporal cortical terminations into the middle auditory cortex (Balezeau et al., 2020). It is, however, not to be excluded that this human-unique feature could be: 1) due to the expansion of the temporal lobe alone (Eichert et al. 2019; Van Essen and Dierker 2007); or 2) due to methodological differences (Latini et al., 2021; Petrides, 2014) (Figure 4).

In their diffusion MRI work, Rilling and colleagues (2008; 2012) described major changes during primate evolution with an increased temporal lobe projection in the human brain and related this finding to language evolution. While the human AF reaches the MTG, the macaque $\mathrm{AF}$ terminates at the caudal end of temporal area 22, near the temporo-parietal junction. As such, Rilling et al. (2008) suggested that the human AF is unique in its extended projections into the middle part of the temporal cortex. This finding has become the basis of a number of models of human language processing. For example, in their model for "Social Communication And Language Evolution and Development" (SCALED), Catani and Bambini (2014) linked the lack of the AF's deep terminations into the middle anterior part of the temporal lobe (long temporal tail) in NHPs to the absence of syntax processing and reduced semantics processing.

At the same time, Van Essen and Dieker (2007) also proposed a theory of primate brain evolution that described a cortical expansion across primates using certain key regions of interest as common landmarks. They proposed that if the macaque brain was inflated to the size of a human brain, some areas would be too small in comparison to their human counterparts. Thus, these areas, including the middle temporal cortex, must have expanded disproportionally in size during evolution to match the actual human brain organisation. As such, this temporal cortical surface expansion could explain the apparent expansion of AF terminations into the middle part of the temporal lobe in the human brain, as a by-product of cortical expansion.

By examining the evolution of temporal association fibres across primate species, a series of studies from Rogier Mars' laboratory, using common space comparisons, tackled the question of whether the observed extension of the AF's termination into the human temporal lobe was due to cortical expansion alone, or also to an actual extension of the tract into new cortical regions (Bryant et al.; 2020; Eichert et al., 2019; 2020; Mars et al., 2021; Roumazeilles et al., 2020; Warrington et al, 2020).

In a similar approach to Van Essen and Dieker (2007), Eichert et al., (2019) first calculated a common space for macaques and humans, based on cortical areas. In a second step, they extracted the main AF tract body (based on Schmahmann and Pandya (2006) atlas - which did not depict the AF's termination into the inferior frontal cortex) using common protocols between species (Warrington et al, 2020) and computed surface projections from these tracts by creating tract maps that show where the tract projected to on the surface. Finally, they tested 
whether the cortical area comparison alone would explain the AF cortical projections. In other words, if a predicted tract map is greater than the actual tract map, than cortical surface expansion does not fully account for the observed expansion of the particular tract (see also Eichert et al., (2020) for a similar method based on T1w/T2w maps alignments). This way, they highlight that cortical expansion alone cannot account for the AF expansion (Eichert et al. 2019; 2020). The authors therefore conclude that the AF underwent additional changes in its course by invading new middle and anterior temporal areas. Thus, the authors propose that the temporal lobe and especially the middle temporal gyrus (MTG) expanded significantly in the great ape lineage. Finally, in the evolution of the human lineage, the AF invaded new territories of the temporal cortex, which made cognitive changes possible (Roumazeilles et al., 2020). Confirming these findings, Barrett et al. (2020) found no middle temporal terminations to connect with frontal areas in macaque ex-vivo dMRI data as in humans. They state that the AF shows the most striking differences of association tracts between humans and monkeys, with monkeys only sharing a small subcomponent of the AF (roughly the body of the tract) with humans.

Even though other diffusion MRI investigations on monkeys have found AF temporal terminations more anterior in the temporal lobe (Schmahmann and Pandya, 2007; Balezeau et al., 2020; Rocchi et al., 2021) than previously shown by tract tracing (e.g., Schmahmann and Pandya, 2006), the monkey's AF's temporal tail is still incomparable with that of the human, where it exceeds the middle part of the temporal lobe. However, very recent studies found for the first-time connections between the functionally defined auditory cortex and Broca's homologue. The auditory cortex areas, which sit in the middle and posterior temporal lobe showed connections with Broca's homologue in the monkeys (Balezeau et al., 2020; Rocchi et al., 2021). Because of this newly highlighted extension into the auditory cortex, the authors speculate "that this dorsal auditory pathway is involved in not just spatial processing in the classical sense but also sound and vocal patterning in the time domain", a function that had previously been exclusively linked to the ventral pathway (see section: "Dorsal vs Ventral Pathway proportion"). Note that in this description, the human AF also terminates in the posterior temporal lobe and does not exceed into the middle part of the temporal lobe.

In fact, it is to note that the very fact that the human AF arches into the middle, anterior and inferior part of the temporal lobe, is questioned by some authors (Latini et al. 2021; Maldonado et al. 2013; Petrides 2014; Wang et al. 2013). In fact, in the monkey brain it was demonstrated that monosynaptic AF connections originate around area tpt/Planum Temporale. Fibres arching more ventrally in the temporal lobe (ie. temporo-parietal fibres like the posterior middle longitudinal fasciculus (MdLF)) are independent and build synapses with the AF (Distler et al. 1993; Petrides 2014; Seltzer and Pandya 1984). This morphology may be similar in the human brain, with the human AF also originating in area tpt/Planum Temporale if defined monosynaptically. However, with diffusion MRI, one is unable to distinguish a monosynaptical $\mathrm{AF}$ originating in area tpt/Planum Temporale or a polysynaptical AF entanglement with the posterior MdLF. In other words, in this view, the AF is losing its predominant "arch" (which corresponds to the posterior indirect segment (Catani et al., 2005)), due to methodological issues (Figure 3). It is possible that this hypothis influenced the most recent comparative investigations (Balzeau et al., 2020; Rocchi et al., 2021). Indeed, in these studies the human AF is terminating more posteriorly and less ventrally in the temporal lobe than in previous work (Barrett et al., 
2020; Eichert et al., 2019; Rilling et al., 2008; 2012), but terminating like their monkey

\section{Question 3: The Dorsal Language Pathway, relative to the Ventral Pathway, has expanded significantly in humans than NHPs?}

In humans the AF together with the SLF II, III makes up the Dorsal language pathway in opposition to the Ventral pathway (e.g., Saur et al., 2008) (Figure 6). Both pathways are connecting inferior frontal with temporal language areas. The Ventral pathway passing through the extreme capsule curves ventrally around the Sylvian fissure in opposition to the Dorsal pathway which curves dorsally around the Sylvian fissure (e.g., Hickok and Poeppel, 2004). Many diffusion MRI studies cannot account for a clear separation of AF and SLF II, III and have therefore based their interpretations on a mix of these bundles rather than only the AF (e.g., Balezeau et al., 2020; Eichert et al., 2019; Rilling et al., 2008; 2012). Therefore, we also keep here the appellation "Dorsal pathway" as in the original articles (e.g., Rilling et al., 2008; 2012) knowing it includes broadly the AF and SLF II and SLF III. Traditionally, at the functional level, the Dorsal pathway is involved in mapping sound to articulation, while the Ventral pathway is involved in mapping sound to meaning (Hickok and Poeppel, 2004; Saur et al., 2008). While both pathways connect similar areas, it is the Dorsal/AF pathway that is dominant in humans (i.e., stronger structural connectivity than the Ventral pathway) (Rilling et al., 2008). Therefore, a crucial question is whether the same organisation is shared with monkeys, or whether actually the Ventral pathway is dominant over the Dorsal pathway in monkeys.

Currently, there are several theoretical accounts based on how a reduced or inversed Dorsalversus Ventral dominance in nonhuman primates could explain the uniqueness of human language (e.g., Friederici, 2017; Pulvermueller, 2018; Rilling et al., 2008; 2012). However, with differences in seeding and the addition of functional connectivity, recent investigations have challenged this view and show increased connectivity on the structural side for the dorsal pathway and an equal dominance of both pathways on the functional side in monkeys (Balezeau et al., 2020; Rocchi et al., 2021). Here, we review tractography and effective connectivity studies of the Dorsal and Ventral pathways to investigate whether or not a stronger ventral-than-dorsal ratio exists in the nonhuman primate's brain.

In their pioneering work, Rilling and colleagues (2008) compared the Dorsal and Ventral pathway across primate species, using diffusion MRI and a probabilistic multi-fibre diffusion model. They found a stronger (more streamlines) Dorsal-than Ventral Pathway in human, which was not the case in macaques and chimpanzees. In fact, for macaques a very weak (i.e., low 
number of streamlines) Dorsal pathway was described, where frontal terminations were found in area 46 and delicately in area 44 (part of Broca's homologue). Temporal terminations were described only in the most posterior end of the temporal lobe in the junction with the parietal lobe. In contrast, a strong Ventral pathway was highlighted with terminations in area 44 and 45 (Broca's homologue) as well as in the frontal operculum and across the temporal lobe (see Figure 4, left panel). In humans the authors described an opposite organisation with a Dorsal pathway stronger than the Ventral one, connecting both similar areas (Rilling et al., 2008). In addition, in comparisons to chimpanzees, they described qualitatively stronger connections to Broca's area (area 44 and 45) via the Dorsal pathway, than via the Ventral pathway (Rilling et al., 2012) in humans. The authors concluded that the Dorsal pathway changed significantly more than the Ventral pathway in human evolution, due to the overall strengthening, greater strength than the Ventral pathway, stronger area 44 and new area 45 terminations as well as extended terminations into (and beyond) the middle part of the temporal lobe (replicated e.g., by Eichert et al., 2019) (Figure 6 left panel). The authors further conclude that the proportional strengthening of the Dorsal Pathway might have contributed to transfer lexical-semantic information and some elements of syntax in the evolution of human language. In contrasts, its monkey's homologue would be rather involved in low-level spatial processing of sounds (Aboitiz, 2018; Rilling et al., 2008), similar to the view of Schmahmann \& Pandya (2006) (Question 1 above). Also, the great extent of temporal lobe information like auditory information would be exclusively conveyed via the ventral pathway in monkeys (in agreement with functional and tract tracing results (Romanski et al. 1999)). This finding and interpretation had a conceptual impact on several theories trying to retrace the evolution of human language. Here we will focus on two of them (Friederici, 2017; Pulvermueller, 2018).

A first prominent theory comes from Angela Friederici as highlighted in her 2017 book "Language in the brain" (Friederici, 2017). She proposes that complex syntax and therefore human language developed in human only, due to the strengthening of the Dorsal pathway with extension of terminations into area 44 (part of Broca's area). The reasoning is the following: The Dorsal pathway conveys complex syntax in the human brain due to the connection to area 44 (Friederici et al. 2006), whereas the Ventral pathway conveys semantic aspects of language and simple syntax due to the connection to area 45 and the frontal operculum (Friederici, 2017). Monkeys however show non or only weak dorsal connections to area 44 (Rilling et al., 2008), but have ventral termination to this area. Therefore, they don't show complex syntax, but aspects of semantics and simple syntax (Friederici, 2017). Indeed, behavioural data indicates that monkeys fail to show the processing of complex syntax, but showed aspects of semantics (Friederici, 2017). In addition, behavioural data in developmental studies highlight that infants only acquire complex syntax by the age of seven, exactly when the most anterior portion of the Dorsal pathway matures and connects to Broca's area (Brauer et al. 2013; Brauer et al. 2011; Friederici 2017; Perani et al. 2011). In contrast, the Ventral pathway connection into this area is already present at that age (Brauer et al. 2013; Brauer et al. 2011; Friederici 2017; Perani et al. 2011).

42 However, there are also some limitations to this theory:

43 First, several diffusion MRI studies interpret the developmental data differently (Dubois et al. 44 2009, 2016). In their view Broca's Dorsal pathway terminations are present before the age of 7 , 45 but intractable in diffusion MRI due to their immature microstructure. Second, more recent 46 behavioural studies highlight that monkey can "break to the syntax barrier" (Ferrigno et al. 2020; 
Jiang et al. 2018; Malassis et al., 2020; Fitch 2018).

A second recent and influential theory based on a weaker Dorsal pathway in NHP was proposed by Pulvermueller (2018). He hypothesised that the overall strengthening, and the evolutionary new and stronger area 44 termination of the Dorsal pathway enabled frontotemporal sensorimotor shortcuts, connecting further than just next neighbouring areas. This way, humans can better bind action and perception into circuits that support cognitive functions important for language processing, like working memory, prediction, attention, and combination, including abstract rule formation. In this work, action representations lie at the heart of language processing.

Very recently however, changes in techniques and seeds for tracking have challenged the view of a stronger-Ventral-than-Dorsal-pathway in monkeys. In a diffusion MRI studies across primate species, Balezeau and colleagues (2020) used a functionally defined auditory cortex as the seed for tractography. This way, they highlighted a Dorsal pathway connection into the auditory cortex, which was until then reserved to the Ventral pathway (Rilling et al., 2008; Romanski et al, 1999). The authors therefore suggest that the monkey AF is not solely involved in low-level processes but instead important for sound and vocal patterning in the time domain (Balezeau et al., 2020).

Furthermore, on the functional level, using effective connectivity while stimulating the same auditory cortex, Rocchi and colleagues (2021) reported that Dorsal and Ventral pathways connect to the same degree to Broca's homologue (area 44 and 45) and the frontal operculum. In other words, even though the Ventral pathway was shown stronger on a structural level (Rilling et al., 2008; 2012), functionally both pathways have the same strength. The authors suggest therefore that language abilities allowing humans to name, conceptualize and thus better remember sound (Schulze et al., 2012) would be shared across primates (Figure 6 right panel).

Based on the above recent data, the long-standing bulwark of an inversed pathway dominance that could explain language evolution is challenged. As a result, contemporary theories that explain the uniqueness of human language based on the inverted proportional strength of the dorsal and ventral pathway, should be revised accordingly. While structural differences in strength may persist between the human and the monkey Dorsal pathway, this is not the case functionally. A question that exceeds the current review is to investigate whether structural differences are therefore of importance on other functional levels as investigated by Rocchi et al. (2021). 
Figure 6: Dorsal- versus Ventral Pathway strength (question 3)
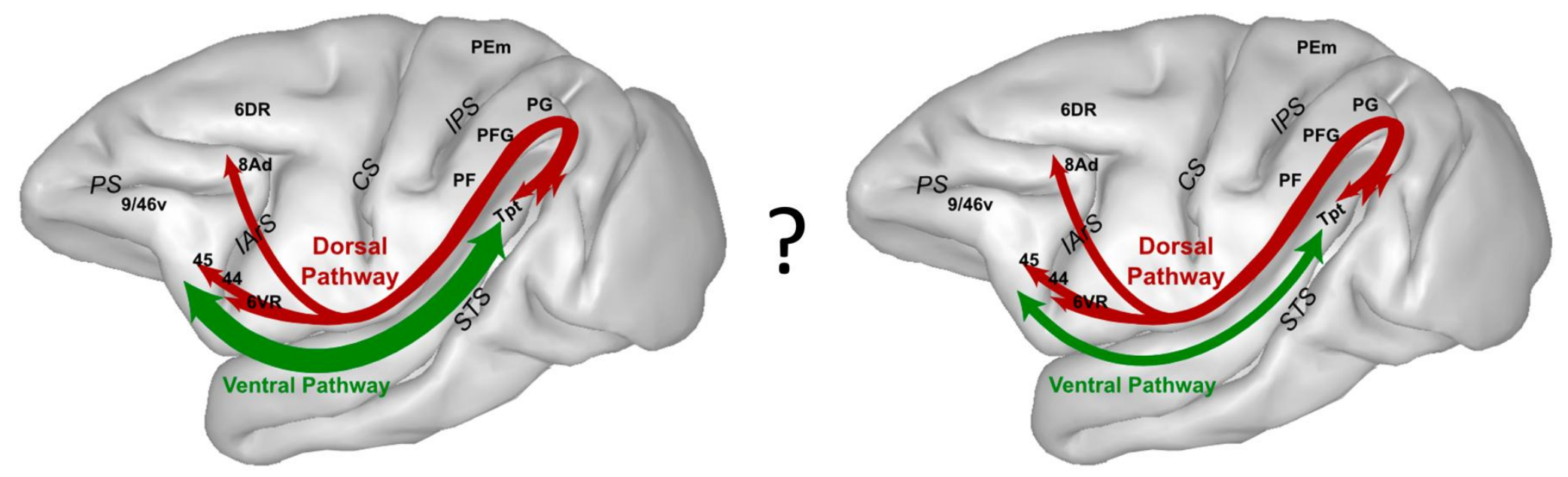

Figure 6: Dorsal- versus Ventral Pathway strength (question 3). (Left) A dominance in strength (in structure and function) in the Ventral pathway (green) against the Dorsal pathway (red) (e.g., Rilling et al., 2008; 2012). (Right) Equal strength (in structure and function) between both

5 pathways (e.g., Rocchi et al., 2021).

In humans, the AF is strongly lateralised towards the left hemisphere (e.g., Thiebaut de Schotten et al., 2011). This structural lateralisation is of interest, because the majority of the language system is also functionally left-lateralised in the brain (e.g., Toga and Thompson 2003). Whether such a same lateralisation exists, or not in nonhuman primates is therefore a crucial question that would shed light on the origins of human language lateralisation. Especially, since the most recent investigations about the AF's anatomy across primates agree on a highly evolutionarily conserved anatomy (see sections above). Thus, the question about anatomical differences across primates, that might explain the human uniqueness for language, persists. Authors of recent investigations highlight that the left AF lateralisation is the crux of the human-specific distinction (Balezeau et al., 2020; Eichert et al., 2019; Rocchi et al., 2021). While carefully reviewing the recent comparative brain lateralisation data, we conclude that this compelling idea lacks sufficient sample size.

From early human development on, inter-hemispheric asymmetry was documented for most language-related regions at both structural and functional level. In fact, language tasks elicit greater activation in the left- than in the right hemisphere, even in newborns (e.g., DehaeneLambertz et al. 2002). Structural markers of such language brain lateralisation were proposed, like the leftward lateralisation of the Planum Temporale, Broca's area or the Insula (e.g., Geschwind and Levitsky 1968; Keller et al. 2010). 
structural markers not only in apes (e.g., Hopkins et al. 2015) but also in an Old World monkey, the baboon, in both adults and newborns (Becker et al. 2021a; b; c; Marie et al. 2018).

3 Regarding white matter in humans, volume of the SLF II and SLF III was shown larger in the right hemisphere (e.g., Amemiya et al. 2021; Thiebaut de Schotten et al. 2011), which is a phylogenetically shared feature with chimpanzees (Hecht et al. 2015). In contrast, the human AF was shown larger in the left hemisphere for a number of macroscopic and microscopic measurements like the number of streamlines, volume of the tract, fibre density and mean fractional anisotropy in adults and infants (Catani et al. 2007; Dubois et al. 2009; Nucifora et al. 2005; Powell et al. 2006; Thiebaut de Schotten et al. 2011; Takaya et al. 2015; Thiebaut de Schotten et al. 2011). Therefore, the structural AF asymmetry, and specifically its temporal terminations, is indeed discussed as a marker for the functional lateralisation of the language network (e.g.,, Catani et al., 2007; Powell et al., 2006; Takaya et al., 2015; but see also e.g., Silva and Citterio 2017; Vernooij et al. 2007) and was further supported by resting state MRI data showing functional lateralisation of the language network (Zhu et al. 2014). However, Verhelst et al. (2021) recently reported that the AF is left-lateralised, regardless of left or rightwards language lateralisation.

But what is known about the nonhuman primate's AF lateralisation? Initially, Rilling et al. (2008) did not find any AF asymmetry in three macaque and four chimpanzee subjects. A follow-up study in chimpanzees including a higher number of subjects (Rilling et al., 2012), showed a left-lateralised AF for 26 subjects. However, this lateralisation in chimpanzee was weaker than in humans. Eichert et al., (2019) confirmed an absence of left asymmetry for the AF/Dorsal pathway in four ex-vivo macaque with high resolution and one invivo macaque image. In contrast, a strong left lateralisation was reported for the Ventral pathway, which was found slightly right lateralised in humans (Eichert et al., 2019). Similarly, Balezeau et al., (2020) reported no lateralisation for the Dorsal pathway in three macaques but also no lateralisation for the three chimpanzee subjects. This latter finding was confirmed on a functional level by Rocchi et al. (2021) for human and macaque subjects. Despite only treating two macaque subjects, a phylogenetical discontinuity in lateralisation was highlighted, with humans being leftwards lateralised and monkeys showing no lateralisation.

As one can easily see, studies that investigated lateralisation produced inconsistent results and are limited to small sample sizes. Indeed, it is common in neuroscience to use not more than two macaque individuals in a given study, which makes interpretation of a populational level lateralisation impossible. Tract tracing methods, for example, are not able to give a response to the questions, while diffusion MRI tractography may do. A small sample-size also limits homogeneous groups in regards to handedness, which was shown to be linked to brain lateralisation (e.g., Hopkins et al., 2015; Meguerditchian et al., 2013), and which might influence therefore influence a population -level lateralisation.

In addition, while lateralisation of homologous perisylvian language areas were found present in several ape species (i.e., Hopkins et al., 2015), these asymmetries were not detected in macaques (Lyn et al., 2011), which are commonly representing the family of Old world monkeys. In contrast, recent studies demonstrated that another Old world monkey, the baboon, possesses homologous asymmetries (Becker et al., 2021a; c; Marie et al., 2018). It remains unclear why cerebral asymmetries are commonly detected in baboons but not in macaques. 
Based on the above tractography data, the question about evolutionary continuities or discontinuities in AF lateralisation will need further investigations with 1) ample sample sizes also in regard to handedness and 2) the inclusion of the baboon as a representative of the Old world monkey family. Therefore, we predict that future studies will focus on this crucial question in language brain evolution.

The apparent literature about the evolution of the AF has been rapidly gaining in interest in very recent years and is used as a base for theories about language evolution. However, no clear consensus was found, due to differences in appellations and divergent anatomical courses and terminations, which are resulting in diverging definitions of what exactly the $\mathrm{AF}$ is.

For example, anatomically, AF and SLF are generally referred to the same tract. This is important as the label "AF" implies "language pathway" more than the label SLF, MdLF or Dorsal pathway. But note that all terms are used interchangeably throughout the literature. However, differences in terminology matters, because a same result could have different theoretical implications depending on its labelling and therefore its functional connotation.

To illustrate this point we can do a thought experiment: If, during primate evolution, an increase of connectivity strength between the superior temporal lobe (Wernicke's area) and the inferior parietal lobe (Geschwind's region) (as in e.g., Aboitiz and García, 1997; Catani and Bambini, 2014) was documented, different functional interpretations could be drawn from this, depending on terminology. We could label this segment for example AF, SLF, MdLF or Dorsal pathway. However, the functional implication would be the most associated with language evolution for the label "AF", even though the segment is the same.

In this review, we have therefore carefully highlighted the history of AF anatomy across primate species and defined a clear current anatomical model. The AF/SLF complex can be divided into four distinct fibre bundles including: 1) the AF, which comprise in itself three fibre branches that arch dorsally around the Sylvian fissure and connect the posterior temporal cortex (area Tpt, posterior auditory cortex) with the frontal cortex (area 44, 45, area 8Ad and 6) and 2) the SLF I, II and III, which comprise the three longitudinal fibre bundles that interconnect distinct parts of the frontal and parietal cortex and are not arching around the Sylvian fissure.

In addition, we focused on four discontinuous key changes in AF anatomy that are discussed to differentiate the human from the nonhuman primate. These are grouped in research questions concerning: frontal terminations (1), temporal terminations (2), the proportion of the Dorsalversus Ventral pathway strength (3) and lateralisation (4). These four key questions are of high importance, because their results in (dis)continuity influence theories about language evolution. Based on the latest results of these four key questions, we argue that the evolution of the AF anatomy is more continuous across primate species than previously described.

When summarizing the four questions (see also Table 1), we can notice the importance of methodological changes that are pivotal in the conceptualisation of the AF anatomy: 
- Historically, knowledge about the AF's terminations (Question 1) relies strongly on invasive tract tracing studies in the monkey brain. Findings about differences in frontal termination in the macaque brain (no Broca's terminations) compared to the human brain (Broca's termination) led to theories about the specific frontal development of the human AF, important for language processing. However, human-like terminations into Broca's homologue in monkeys were found when cytoarchitectonic equivalence was demonstrated and tract tracing injection sites changed. Therefore, the frontal termination's organisation seems to be conserved during primate evolution, even though terminations might have become overall stronger in humans.

- The same applies to the temporal terminations (Question 2), which were initially found to be very extended in humans, exceeding the middle part of the temporal lobe into its anterior and inferior parts. New data from methodological changes indicate however, that first, the monkey's terminations are also slightly more extended (tracking from the functionally characterised auditory cortex) and second, the human extension could be a technical artefact (possible entanglement with MdLF fibres). Therefore, it is not excluded that the temporal termination's organisation might also be conserved during primate evolution, even though the temporal connections might have become overall stronger in humans.

- Finally, pivotal changes in the question of the proportion of the Ventral- versus the Dorsal pathway's strength (Question 3), can also be illustrated by changes in techniques (effective connectivity) and seed masks (tracking from the functionally characterised auditory cortex). Initially found to be very weak and not terminating into the auditory cortex, the Dorsal pathway in monkeys is now demonstrated to be functionally as strong as the Ventral pathway and also connecting with the auditory cortex. Therefore, the Ventral-versus-Dorsal pathway strength organisation seems to be conserved during primate evolution, even though the Dorsal pathway might have become overall structurally stronger in humans.

- Using this method-based framework on the question of lateralisation (Question 4), we can say that currently only the progress of tractography methods and dMRI acquisition techniques could advance this question as tract-tracing methods are not adapted. In particular, a forthcoming development of tractography applied to a large-scale cohort of non-human primate data could further advance the question as it was already conducted in chimpanzees concerning the lateralisation of other tracts like the SLF (Hecht et al., 2015), or structural grey matter asymmetries (e.g., Hopkins et al., 2015). Therefore, the question of AF lateralisation across primates needs a specific attention in the future. Given existing results about the leftlateralisations of perisylvian regions in monkeys (Becker et al., 2021a; Marie et al., 2018), it is not excluded that a human-like left lateralisation could also be demonstrated in monkeys in the future, even though lateralisation might have become stronger in human evolution.

In addition, future studies must also focus on other tracts that might have undergone substantial change during primate evolution and that are linked to language processing in humans. Possible tracts of interest are the Ventral pathway (and its subcomponents), the middle longitudinal fasciculus and the frontal aslant (for a review: La Corte et al., 2021).

In conclusion, recent works in humans and nonhuman primates suggest that the AF course and terminations are more similar between species than described before. The remarkable human ability of language might therefore have evolved progressively in primate evolution, exactly as its underlying anatomic implementation in the brain. Or to put it in the words of Charles Darwin: 
1 is one of degree and not of kind." (page 105: Darwin, 1871/1981). Future theories about

2 language brain evolution should account for this continuous, and not discrete AF evolution.

3

\begin{tabular}{|c|c|c|c|c|}
\hline & \multicolumn{2}{|c|}{ Tract tracer studies } & \multicolumn{2}{|c|}{ Diffusion MRI } \\
\hline & Absent & Present & Absent & Present \\
\hline $\begin{array}{l}\text { Broca's } \\
\text { terminations } \\
(\text { monkey })\end{array}$ & $\begin{array}{c}\text { Petrides \& Pandya } 1988 \\
\text { Schmahmann \& Pandya } \\
2006 \\
\text { Thiebaut de Schotten et } \\
\text { al., } 2012\end{array}$ & $\begin{array}{c}\text { Petrides \& Pandya } \\
2009 \\
\text { Frey et al., } 2014 \\
\text { Petrides et al., } 2014\end{array}$ & l & $\begin{array}{c}\text { Rilling et al., } 2008 \\
\text { Eichert et al., 2019; } 2020 \\
\text { Barrett et al., } 2020 \\
\text { Balezeau et al., } 2020 \\
\text { Bryant et al., } 2020 \\
\text { Roumazeilles et al., } 2020\end{array}$ \\
\hline $\begin{array}{c}\text { Auditory } \\
\text { cortex } \\
\text { terminations } \\
\text { (temporal } \\
\text { lobe) } \\
\text { (monkey) }\end{array}$ & $\begin{array}{c}\text { Schmahmann \& Pandya } \\
2006 \\
\text { Petrides \& Pandya } 2009 \\
\text { Frey et al., } 2014 \\
\text { Petrides et al., } 2014\end{array}$ & / & $\begin{array}{c}\text { Eichert et al., } 2019 ; 2020 \\
\text { Barrett et al., } 2020 \\
\text { Roumazeilles et al., } 2020 \\
\text { Rilling et al., } 2008 ; 2012\end{array}$ & $\begin{array}{c}\text { Balezeau et al., } 2020 \\
\text { Rocchi et al., } 2021\end{array}$ \\
\hline $\begin{array}{c}\text { Extended } \\
\text { temporal lobe } \\
\text { terminations } \\
\text { (human) }\end{array}$ & / & / & $\begin{array}{l}\text { Maldonando et al., 2013; } \\
\text { Wang et al., 2013; } \\
\text { Petrides, 2014; } \\
\text { Balezeau et al., 2020 } \\
\text { Barbeau et al. ; } 2020 \\
\text { Latini et al., 2021 }\end{array}$ & $\begin{array}{c}\text { Catani et al., } 2005 \\
\text { Rilling et al., } 2008 \\
\text { Thiebaut de Schotten et } \\
\text { al., } 2012 \\
\text { Eichert et al., } 2019\end{array}$ \\
\hline $\begin{array}{c}\text { Ventral > } \\
\text { Dorsal } \\
\text { Pathway } \\
\text { (monkey) }\end{array}$ & / & I & Rocchi et al., 2021 & Rilling et al., $2008 ; 2012$ \\
\hline $\begin{array}{c}\text { Lateralisation } \\
\text { (chimpanzee) }\end{array}$ & / & / & Balezeau et al., 2020 & Rilling et al., $2008 ; 2012$ \\
\hline $\begin{array}{l}\text { Lateralisation } \\
\quad(\text { monkey })\end{array}$ & / & / & $\begin{array}{c}\text { Rilling et al., } 2008 ; 2012 \\
\text { Eichert et al., } 2019 \\
\text { Balezeau et al., } 2020 \\
\text { Rocchi et al., } 2021\end{array}$ & / \\
\hline
\end{tabular}

7 Table 1.: Summary of the main work analysed in the present review, in regard to the contrasting 8 descriptions of the Arcuate Fasciculus (AF) in tract-tracer and diffusion MRI studies. Six 9 features are highlighted as absent or present: Broca's terminations in monkeys, temporal 10 terminations into the monkeys' auditory cortex in the temporal lobe, expanded terminations into 11 the human's inferior or anterior temporal lobe, dominance of the Ventral pathway over the 12 Dorsal pathway in monkeys, lateralisation in chimpanzee brains and lateralisation in monkey 13 brains. "/": No data available. 
References:

3
Aboitiz, Francisco, and Ricardo García V. 1997. 'The Evolutionary Origin of the Language Areas in the Human Brain. A Neuroanatomical Perspective'. Brain Research Reviews 25(3):381-96. doi: 10.1016/S0165-0173(97)00053-2.

Amemiya, Kaoru, Eiichi Naito, and Hiromasa Takemura. 2021. 'Age Dependency and Lateralization in the Three Branches of the Human Superior Longitudinal Fasciculus'. Cortex 139:116-33. doi: 10.1016/j.cortex.2021.02.027.

Balezeau, Fabien, Benjamin Wilson, Guillermo Gallardo, Fred Dick, William Hopkins, Alfred Anwander, Angela D. Friederici, Timothy D. Griffiths, and Christopher I. Petkov. 2020. 'Primate Auditory Prototype in the Evolution of the Arcuate Fasciculus'. Nature Neuroscience. doi: 10.1038/s41593-020-0623-9.

Barbeau, Elise B., Maxime Descoteaux, and Michael Petrides. 2020. 'Dissociating the White Matter Tracts Connecting the Temporo-Parietal Cortical Region with Frontal Cortex Using Diffusion Tractography'. Scientific Reports 10(1):8186. doi: 10.1038/s41598-020-64124-y.

Barrett, Rachel L. C., Matthew Dawson, Tim B. Dyrby, Kristine Krug, Maurice Ptito, Helen D'Arceuil, Paula L. Croxson, Philippa J. Johnson, Henrietta Howells, Stephanie J. Forkel, Flavio Dell' Acqua, and Marco Catani. 2020. 'Differences in Frontal Network Anatomy Across Primate Species'. Journal of Neuroscience 40(10):2094-2107. doi: 10.1523/JNEUROSCI.1650-18.2019.

Becker, Yannick, Konstantina Margiotoudi, Damien Marie, Muriel Roth, Bruno Nazarian, Romain Lacoste, Jean-Luc Anton, Olivier Coulon, Nicolas Claidiere, and Adrien Meguerditchian. 2021. 'On the Gestural Origin of Language Lateralisation: Manual Communication Reflects Broca's Asymmetry in Monkeys'. BioRxiv 2021.02.09.430276. doi: 10.1101/2021.02.09.430276.

Becker, Yannick, Romane Phelipon, Julien Sein, Lionel Velly, Luc Renaud, and Adrien Meguerditchian. 2021. 'Planum Temporale Grey Matter Volume Asymmetries in Newborn Monkeys (Papio Anubis)'. Brain Structure and Function. doi: 10.1007/s00429-021-02278-9.

Becker, Yannick, Julien Sein, Lionel Velly, Laura Giacomino, Luc Renaud, Romain Lacoste, Jean-Luc Anton, Bruno Nazarian, Cammie Berne, and Adrien Meguerditchian. 2020. 'Early Left-Planum Temporale Asymmetry in Newborn Monkeys (Papio Anubis): A Longitudinal Structural MRI Study at Two Stages of Development'. NeuroImage 117575. doi: 10.1016/j.neuroimage.2020.117575.

Binder, Jeffrey R., Rutvik H. Desai, William W. Graves, and Lisa L. Conant. 2009. 'Where Is the Semantic System? A Critical Review and Meta-Analysis of 120 Functional Neuroimaging Studies'. Cerebral Cortex 19(12):2767-96. doi: 10.1093/cercor/bhp055.

Brauer, Jens, Alfred Anwander, and Angela D. Friederici. 2011. 'Neuroanatomical Prerequisites for Language Functions in the Maturing Brain'. Cerebral Cortex 21(2):459-66. doi: 10.1093/cercor/bhq108. 
Brauer, Jens, Alfred Anwander, Daniela Perani, and Angela D. Friederici. 2013. 'Dorsal and Ventral Pathways in Language Development'. Brain and Language 127(2):289-95. doi: 10.1016/j.bandl.2013.03.001.

Bryant, Katherine L., Longchuan Li, and Rogier B. Mars. 2020. A Comprehensive Atlas of White Matter Tracts in the Chimpanzee. PLoS biology, 18(12) e3000971

Burdach, Karl Friedrich. 1826. Vom Baue und Leben des Gehirns. Dyk.

Catani, Marco, Matthew P. G. Allin, Masud Husain, Luca Pugliese, Marsel M. Mesulam, Robin M. Murray, and Derek K. Jones. 2007. 'Symmetries in Human Brain Language Pathways Correlate with Verbal Recall'. Proceedings of the National Academy of Sciences 104(43):17163-68. doi: 10.1073/pnas.0702116104.

Catani, Marco, and Valentina Bambini. 2014. 'A Model for Social Communication And Language Evolution and Development (SCALED)'. Current Opinion in Neurobiology 28:165-71. doi: 10.1016/j.conb.2014.07.018.

Catani, Marco, Robert J. Howard, Sinisa Pajevic, and Derek K. Jones. 2002. 'Virtual in Vivo Interactive Dissection of White Matter Fasciculi in the Human Brain'. NeuroImage 17(1):77-94. doi: 10.1006/nimg.2002.1136.

Catani, Marco, Derek K. Jones, and Dominic H. ffytche. 2005. 'Perisylvian Language Networks of the Human Brain'. Annals of Neurology 57(1):8-16. doi: 10.1002/ana.20319.

Catani, Marco, and Marsel Mesulam. 2008. 'The Arcuate Fasciculus and the Disconnection Theme in Language and Aphasia: History and Current State'. Cortex 44(8):953-61. doi: 10.1016/j.cortex.2008.04.002.

Catani, Marco, and Michel Thiebaut de Schotten. 2012. Atlas of Human Brain Connections. OUP Oxford.

Damasio, Antonio R., and Norman Geschwind. 1984. 'The Neural Basis of Language'. Annual Review of Neuroscience 7(1):127-47. doi: 10.1146/annurev.ne.07.030184.001015.

Darwin, Charles. 1981. 'The descent of man and selection in relation to sex (Vol. 1)'. Princeton University press (Originally work published 1871).

Dehaene-Lambertz, Ghislaine, Stanislas Dehaene, and Lucie Hertz-Pannier. 2002. 'Functional Neuroimaging of Speech Perception in Infants'. Science 298(5600):2013-15. doi: 10.1126/science. 1077066 .

Dejerine, J., Jules and Augusta Dejerine. 1895. Anatomie des centres nerveux. Rueff.

Dick, Anthony Steven, and Pascale Tremblay. 2012. 'Beyond the Arcuate Fasciculus: Consensus and Controversy in the Connectional Anatomy of Language'. Brain 135(12):3529-50. doi: 10.1093/brain/aws222.

Distler, Claudia, Boussaoud, Driss, Desimone Robert, and Leslie G. Ungerleider. 1993. 'Cortical Connections of Inferior Temporal Area TEO in Macaque Monkeys'. Journal of Comparative Neurology 334(1):125-50. doi: 10.1002/cne.903340111. 
Dubois, Jessica, Hertz-Pannier, Lucie, Cachia, Arnaud, Mangin, Jean-Francois, Le Bihan, Denis, and Dehaene-Lambertz, Ghislaine. 2009. 'Structural Asymmetries in the Infant Language and Sensori-Motor Networks’. Cerebral Cortex 19(2):414-23. doi: 10.1093/cercor/bhn097.

Dubois, Jessica, Cyril Poupon, Bertrand Thirion, Hina Simonnet, Sofya Kulikova, François Leroy, Lucie Hertz-Pannier, and Ghislaine Dehaene-Lambertz. 2016. 'Exploring the Early Organization and Maturation of Linguistic Pathways in the Human Infant Brain'. Cerebral Cortex 26(5):2283-98. doi: 10.1093/cercor/bhv082.

Eichert, Nicole, Emma C. Robinson, Katherine L. Bryant, Saad Jbabdi, Mark Jenkinson, Longchuan Li, Kristine Krug, Kate E. Watkins, and Rogier B. Mars. 2020. 'Cross-Species Cortical Alignment Identifies Different Types of Anatomical Reorganization in the Primate Temporal Lobe' edited by T. Verstynen, J. I. Gold, T. Verstynen, and K. Heuer. ELife 9:e53232. doi: 10.7554/eLife.53232.

Eichert, Nicole, Lennart Verhagen, Davide Folloni, Saad Jbabdi, Alexandre A. Khrapitchev, Nicola R. Sibson, Dante Mantini, Jerome Sallet, and Rogier B. Mars. 2019. 'What Is Special about the Human Arcuate Fasciculus? Lateralization, Projections, and Expansion'. Cortex 118:107-15. doi: 10.1016/j.cortex.2018.05.005.

Fernández-Miranda, Juan C., Albert L. Rhoton Jr., Juan Álvarez-Linera, Yukinari Kakizawa, Chanyoung Choi, and Evandro P. de Oliveira. 2008. 'Three-Dimensional Microsurgical and Tractographic Anatomy of the White Matter of the Human Brain'. Neurosurgery 62:SHC989-1028. doi: 10.1227/01.NEU.0000297076.98175.67.

Ferrigno, Stephen, Samuel J. Cheyette, Steven T. Piantadosi, and Jessica F. Cantlon. 2020. 'Recursive Sequence Generation in Monkeys, Children, U.S. Adults, and Native Amazonians'. Science Advances 6(26):eaaz1002. doi: 10.1126/sciadv.aaz1002.

Frey, Stephen, Jennifer S. W. Campbell, G. Bruce Pike, and Michael Petrides. 2008. 'Dissociating the Human Language Pathways with High Angular Resolution Diffusion Fiber Tractography'. Journal of Neuroscience 28(45):11435-44. doi: 10.1523/JNEUROSCI.2388-08.2008.

Frey, Stephen, Scott Mackey, and Michael Petrides. 2014. 'Cortico-Cortical Connections of Areas 44 and 45B in the Macaque Monkey'. Brain and Language 131:36-55. doi: 10.1016/j.bandl.2013.05.005.

Friederici, Angela D. 2017. Language in Our Brain: The Origins of a Uniquely Human Capacity. Cambridge, Massachusetts: The MIT Press.

Friederici, Angela D., Jörg Bahlmann, Stefan Heim, Ricarda I. Schubotz, and Alfred Anwander. 2006. 'The Brain Differentiates Human and Non-Human Grammars: Functional Localization and Structural Connectivity'. Proceedings of the National Academy of Sciences 103(7):2458-63. doi: 10.1073/pnas.0509389103.

Friedrich, Patrick, Stephanie J. Forkel, Céline Amiez, Joshua H. Balsters, Olivier Coulon, Lingzhong Fan, Alexandros Goulas, Fadila Hadj-Bouziane, Erin E. Hecht, Katja Heuer, Tianzi Jiang, Robert D. Latzman, Xiaojin Liu, Kep Kee Loh, Kaustubh R. Patil, Alizée Lopez-Persem, Emmanuel Procyk, Jerome Sallet, Roberto Toro, Sam Vickery, Susanne Weis, Charles R. E. Wilson, Ting Xu, Valerio Zerbi, Simon B. Eickoff, Daniel S. Margulies, Rogier B. Mars, and Michel Thiebaut 
de Schotten. 2021. 'Imaging Evolution of the Primate Brain: The next Frontier?' NeuroImage 228:117685. doi: 10.1016/j.neuroimage.2020.117685.

Geschwind, Norman. 1970. 'The Organization of Language and the Brain'. Science 170(3961):940-44.

Geschwind, Norman, and Walter Levitsky. 1968. 'Human Brain: Left-Right Asymmetries in Temporal Speech Region'. Science 161(3837):186-87. doi: 10.1126/science.161.3837.186.

Girard, Gabriel, Roberto Caminiti, Alexandra Battaglia-Mayer, Etienne St-Onge, Karen S. Ambrosen, Simon F. Eskildsen, Kristine Krug, Tim B. Dyrby, Maxime Descoteaux, Jean-Philippe Thiran, and Giorgio M. Innocenti. 2020. 'On the Cortical Connectivity in the Macaque Brain: A Comparison of Diffusion Tractography and Histological Tracing Data'. Neurolmage 221:117201. doi: 10.1016/j.neuroimage.2020.117201.

Hecht, Erin E., David A. Gutman, Bruce A. Bradley, Todd M. Preuss, and Dietrich Stout. 2015. 'Virtual Dissection and Comparative Connectivity of the Superior Longitudinal Fasciculus in Chimpanzees and Humans'. NeuroImage 108:124-37. doi: 10.1016/j.neuroimage.2014.12.039.

Hickok, Gregory, and David Poeppel. 2004. 'Dorsal and Ventral Streams: A Framework for Understanding Aspects of the Functional Anatomy of Language'. Cognition 92(1):67-99. doi: 10.1016/j.cognition.2003.10.011.

Holland, Scott K., Jennifer Vannest, Marc Mecoli, Lisa M. Jacola, Jan-Mendelt Tillema, Prasanna R. Karunanayaka, Vincent J. Schmithorst, Weihong Yuan, Elena Plante, and Anna W. Byars. 2007. 'Functional MRI of Language Lateralization during Development in Children'. International Journal of Audiology 46(9):533-51. doi: 10.1080/14992020701448994.

Hopkins, William D., Maria Misiura, Sarah M. Pope, and Elitaveta M. Latash. 2015. 'Behavioral and Brain Asymmetries in Primates: A Preliminary Evaluation of Two Evolutionary Hypotheses: Evolution of Asymmetries'. Annals of the New York Academy of Sciences 1359(1):65-83. doi: 10.1111/nyas.12936.

Hula, William D., Sandip Panesar, Michelle L. Gravier, Fang-Cheng Yeh, Haley C. Dresang, Michael Walsh Dickey, and Juan C. Fernandez-Miranda. 2020. 'Structural White Matter Connectometry of Word Production in Aphasia: An Observational Study'. Brain 143(8):2532-44. doi: 10.1093/brain/awaa193.

Jiang, Xinjian, Tenghai Long, Weicong Cao, Junru Li, Stanislas Dehaene, and Liping Wang. 2018. 'Production of Supra-Regular Spatial Sequences by Macaque Monkeys'. Current Biology 28(12):1851-1859.e4. doi: 10.1016/j.cub.2018.04.047.

Keller, Simon S., Neil Roberts, Marta García-Fiñana, Siawoosh Mohammadi, E. Bernd Ringelstein, Stefan Knecht, and Michael Deppe. 2010. 'Can the Language-Dominant Hemisphere Be Predicted by Brain Anatomy?' Journal of Cognitive Neuroscience 23(8):2013-29. doi: 10.1162/jocn.2010.21563.

La Corte, E., Eldahaby, D., Greco, E., Aquino, D., Bertolini, G., Levi, V., Ottenhausen, M., Demichelis, G., Romito, L.M., Acerbi, F., Broggi, M., Schiariti, M.P., Ferroli, P., Bruzzone, M.G., Serrao, G., 2021. The Frontal Aslant Tract: A Systematic Review for Neurosurgical Applications. Frontiers in Neurology 12, 51. https://doi.org/10.3389/fneur.2021.641586 
Lanciego, Jose L., and Floris G. Wouterlood. 2020. 'Neuroanatomical Tract-Tracing Techniques That Did Go Viral'. Brain Structure and Function 225(4):1193-1224. doi: 10.1007/s00429-020-02041-6.

Latini, Francesco, Gianluca Trevisi, Markus Fahlström, Malin Jemstedt, Åsa Alberius Munkhammar, Maria Zetterling, Göran Hesselager, and Mats Ryttlefors. 2021. 'New Insights Into the Anatomy, Connectivity and Clinical Implications of the Middle Longitudinal Fasciculus'. Frontiers in Neuroanatomy 14:610324. doi: 10.3389/fnana.2020.610324.

Lawes, I. Nigel C., Thomas R. Barrick, Vengadasalam Murugam, Natalia Spierings, David R. Evans, Marie Song, and Chris A. Clark. 2008. 'Atlas-Based Segmentation of White Matter Tracts of the Human Brain Using Diffusion Tensor Tractography and Comparison with Classical Dissection'. NeuroImage 39(1):62-79. doi: 10.1016/j.neuroimage.2007.06.041.

Loh, Kep Kee, Petrides, Michael, Hopkins, William, Procyk, Emmanuel, Amiez, Celine, 2017 'Cognitive control of vocalizations in the primate ventrolateral-dorsomedial frontal (VLF-DMF) brain network'. Neurosci. Biobehav. Rev. 82, 32-44.

Loh, Kep Kee, Emmanuel Procyk, Rémi Neveu, Franck Lamberton, William D. Hopkins, Michael Petrides, and Céline Amiez. 2020. 'Cognitive Control of Orofacial Motor and Vocal Responses in the Ventrolateral and Dorsomedial Human Frontal Cortex'. Proceedings of the National Academy of Sciences 117(9):4994-5005. doi: 10.1073/pnas.1916459117.

Lyn, Heidi, Peter Pierre, Allyson J. Bennett, Scott Fears, Roger Woods, and William D. Hopkins. 2011. 'Planum Temporale Grey Matter Asymmetries in Chimpanzees (Pan Troglodytes), Vervet (Chlorocebus Aethiops Sabaeus), Rhesus (Macaca Mulatta) and Bonnet (Macaca Radiata) Monkeys'. Neuropsychologia 49(7):2004-12. doi: 10.1016/j.neuropsychologia.2011.03.030.

Maier-Hein, Klaus H., Peter F. Neher, Jean-Christophe Houde, Marc-Alexandre Côté, Eleftherios Garyfallidis, Jidan Zhong, Maxime Chamberland, Fang-Cheng Yeh, Ying-Chia Lin, Qing Ji, Wilburn E. Reddick, John O. Glass, David Qixiang Chen, Yuanjing Feng, Chengfeng Gao, Ye Wu, Jieyan Ma, Renjie He, Qiang Li, Carl-Fredrik Westin, Samuel Deslauriers-Gauthier, J. Omar Ocegueda González, Michael Paquette, Samuel St-Jean, Gabriel Girard, François Rheault, Jasmeen Sidhu, Chantal M. W. Tax, Fenghua Guo, Hamed Y. Mesri, Szabolcs Dávid, Martijn Froeling, Anneriet M. Heemskerk, Alexander Leemans, Arnaud Boré, Basile Pinsard, Christophe Bedetti, Matthieu Desrosiers, Simona Brambati, Julien Doyon, Alessia Sarica, Roberta Vasta, Antonio Cerasa, Aldo Quattrone, Jason Yeatman, Ali R. Khan, Wes Hodges, Simon Alexander, David Romascano, Muhamed Barakovic, Anna Auría, Oscar Esteban, Alia Lemkaddem, JeanPhilippe Thiran, H. Ertan Cetingul, Benjamin L. Odry, Boris Mailhe, Mariappan S. Nadar, Fabrizio Pizzagalli, Gautam Prasad, Julio E. Villalon-Reina, Justin Galvis, Paul M. Thompson, Francisco De Santiago Requejo, Pedro Luque Laguna, Luis Miguel Lacerda, Rachel Barrett, Flavio Dell'Acqua, Marco Catani, Laurent Petit, Emmanuel Caruyer, Alessandro Daducci, Tim B. Dyrby, Tim Holland-Letz, Claus C. Hilgetag, Bram Stieltjes, and Maxime Descoteaux. 2017. 'The Challenge of Mapping the Human Connectome Based on Diffusion Tractography'. Nature Communications 8(1):1349. doi: 10.1038/s41467-017-01285-x.

Makris, Nikos, David N. Kennedy, Sean McInerney, A. Gregory Sorensen, Ruopeng Wang, Verne S. Caviness Jr, and Deepak N. Pandya. 2005. 'Segmentation of Subcomponents within the Superior Longitudinal Fascicle in Humans: A Quantitative, In Vivo, DT-MRI Study'. Cerebral Cortex 15(6):854-69. doi: 10.1093/cercor/bhh186. 
Malassis, Raphaëlle, Stanislas Dehaene, and Joël Fagot. 2020. 'Baboons ( Papio Papio ) Process a Context-Free but Not a Context-Sensitive Grammar'. Scientific Reports 10(1):1-12. doi: 10.1038/s41598-020-64244-5.

Maldonado, Igor Lima, Nicolas Menjot de Champfleur, Stéphane Velut, Christophe Destrieux, Ilyess Zemmoura, and Hugues Duffau. 2013. 'Evidence of a Middle Longitudinal Fasciculus in the Human Brain from Fiber Dissection'. Journal of Anatomy 223(1):38-45. doi: 10.1111/joa.12055.

Marie, Damien, Muriel Roth, Romain Lacoste, Bruno Nazarian, Alice Bertello, Jean-Luc Anton, William D. Hopkins, Konstantina Margiotoudi, Scott A. Love, and Adrien Meguerditchian. 2018. 'Left Brain Asymmetry of the Planum Temporale in a Nonhominid Primate: Redefining the Origin of Brain Specialization for Language'. Cerebral Cortex 28(5):1808-15. doi: 10.1093/cercor/bhx096.

Mars, Rogier B., Saad Jbabdi, and Matthew F. S. Rushworth. 2021. 'A Common Space Approach to Comparative Neuroscience'. Annual Review of Neuroscience 44(1):null. doi: 10.1146/annurevneuro-100220-025942.

Mars, Rogier B., Jonathan O’Muircheartaigh, Davide Folloni, Longchuan Li, Matthew F. Glasser, Saad Jbabdi, and Katherine L. Bryant. 2019. 'Concurrent Analysis of White Matter Bundles and Grey Matter Networks in the Chimpanzee'. Brain Structure and Function 224(3):1021-33. doi: 10.1007/s00429-018-1817-8.

Matsumoto, Riki, Dileep R. Nair, Eric LaPresto, Imad Najm, William Bingaman, Hiroshi Shibasaki, and Hans O. Lüders. 2004. 'Functional Connectivity in the Human Language System: A CorticoCortical Evoked Potential Study'. Brain 127(10):2316-30. doi: 10.1093/brain/awh246.

Meguerditchian, Adrien, Jacques Vauclair, and William D. Hopkins. 2013. 'On the Origins of Human Handedness and Language: A Comparative Review of Hand Preferences for Bimanual Coordinated Actions and Gestural Communication in Nonhuman Primates'. Developmental Psychobiology 55(6):637-50. doi: 10.1002/dev.21150.

Meynert, Theodor. 1885. Psychiatry; a Clinical Treatise on Diseases of the Fore-Brain Based upon a Study of Its Structure, Functions, and Nutrition. G.P. Putnam's Sons.

Milham, Michael, Christopher I. Petkov, Daniel S. Margulies, Charles E. Schroeder, Michele A. Basso, Pascal Belin, Damien A. Fair, Andrew Fox, Sabine Kastner, Rogier B. Mars, Adam Messinger, Colline Poirier, Wim Vanduffel, David C. Van Essen, Ashkan Alvand, Yannick Becker, Suliann Ben Hamed, Austin Benn, Clementine Bodin, Susann Boretius, Bastien Cagna, Olivier Coulon, Sherif Hamdy El-Gohary, Henry Evrard, Stephanie J. Forkel, Patrick Friedrich, Sean FroudistWalsh, Eduardo A. Garza-Villarreal, Yang Gao, Alessandro Gozzi, Antoine Grigis, Renee Hartig, Takuya Hayashi, Katja Heuer, Henrietta Howells, Dirk Jan Ardesch, Béchir Jarraya, Wendy Jarrett, Hank P. Jedema, Igor Kagan, Clare Kelly, Henry Kennedy, P. Christiaan Klink, Sze Chai Kwok, Robert Leech, Xiaojin Liu, Christopher Madan, Wasana Madushanka, Piotr Majka, AnnMarie Mallon, Kevin Marche, Adrien Meguerditchian, Ravi S. Menon, Hugo Merchant, Anna Mitchell, Karl-Heinz Nenning, Aki Nikolaidis, Michael Ortiz-Rios, Marco Pagani, Vikas Pareek, Mark Prescott, Emmanuel Procyk, Reza Rajimehr, Ioana-Sabina Rautu, Amir Raz, Anna Wang Roe, Román Rossi-Pool, Lea Roumazeilles, Tomoko Sakai, Jerome Sallet, Pamela GarcíaSaldivar, Chika Sato, Stephen Sawiak, Marike Schiffer, Caspar M. Schwiedrzik, Jakob Seidlitz, Julien Sein, Zhi-ming Shen, Amir Shmuel, Afonso C. Silva, Luciano Simone, Nikoloz Sirmpilatze, Julia Sliwa, Jonathan Smallwood, Jordy Tasserie, Michel Thiebaut de Schotten, Roberto Toro, Regis Trapeau, Lynn Uhrig, Julien Vezoli, Zheng Wang, Sara Wells, Bella 
Williams, Ting Xu, Augix Guohua Xu, Essa Yacoub, Ming Zhan, Lei Ai, Céline Amiez, Fabien Balezeau, Mark G. Baxter, Erwin L. A. Blezer, Thomas Brochier, Aihua Chen, Paula L. Croxson, Christienne G. Damatac, Stanislas Dehaene, Stefan Everling, Lazar Fleysher, Winrich Freiwald, Timothy D. Griffiths, Carole Guedj, Fadila Hadj-Bouziane, Noam Harel, Bassem Hiba, Benjamin Jung, Bonhwang Koo, Kevin N. Laland, David A. Leopold, Patrik Lindenfors, Martine Meunier, Kelvin Mok, John H. Morrison, Jennifer Nacef, Jamie Nagy, Mark Pinsk, Simon M. Reader, Pieter R. Roelfsema, David A. Rudko, Matthew F. S. Rushworth, Brian E. Russ, Michael Christoph Schmid, Elinor L. Sullivan, Alexander Thiele, Orlin S. Todorov, Doris Tsao, Leslie Ungerleider, Charles R. E. Wilson, Frank Q. Ye, Wilbert Zarco, and Yong-di Zhou. 2020. 'Accelerating the Evolution of Nonhuman Primate Neuroimaging'. Neuron 105(4):600-603. doi: 10.1016/j.neuron.2019.12.023.

Nucifora, Paolo G. P., Ragini Verma, Elias R. Melhem, Raquel E. Gur, and Ruben C. Gur. 2005. 'Leftward Asymmetry in Relative Fiber Density of the Arcuate Fasciculus'. NeuroReport 16(8):791-94.

Oliveira, João Vítor Miranda Porto de, Antônio Felipe Raquelo-Menegassio, and Igor Lima Maldonado. 2021. 'What's Your Name Again? A Review of the Superior Longitudinal and Arcuate Fasciculus Evolving Nomenclature'. Clinical Anatomy n/a(n/a). doi: 10.1002/ca.23764.

Perani, Daniela, Saccuman, Maria. C., Scifo, Paolo, Anwander, Alfred, Spada, Danilo, Baldoli, Christina, Poloniato, Antonella, Lohmann, Gabriele, and Friederici, Angela, D. 2011. 'Neural Language Networks at Birth'. Proceedings of the National Academy of Sciences 108(38):16056-61. doi: 10.1073/pnas.1102991108.

Petrides, Michael, and Pandya Deepak. 1999. 'Dorsolateral Prefrontal Cortex: Comparative Cytoarchitectonic Analysis in the Human and the Macaque Brain and Corticocortical Connection Patterns'. European Journal of Neuroscience 11(3):1011-36. doi: 10.1046/j.14609568.1999.00518.x.

Petrides, Michael, and Pandya Deepak. 2002. 'Comparative Cytoarchitectonic Analysis of the Human and the Macaque Ventrolateral Prefrontal Cortex and Corticocortical Connection Patterns in the Monkey'. European Journal of Neuroscience 16(2):291-310. doi: 10.1046/j.14609568.2001.02090.x.

Petrides Michael. 2005. 'Lateral Prefrontal Cortex: Architectonic and Functional Organization'. Philosophical Transactions of the Royal Society B: Biological Sciences 360(1456):781-95. doi: 10.1098/rstb.2005.1631.

Petrides, Michael. 2014. Neuroanatomy of Language Regions of the Human Brain. First edition. Amsterdam: Elsevier/AP, Academic Press is an imprint of Elsevier.

Petrides, Michael, Geneviève Cadoret, and Scott Mackey. 2005. 'Orofacial Somatomotor Responses in the Macaque Monkey Homologue of Broca's Area'. Nature 435(7046):1235. doi: 10.1038 /nature 03628 .

Powell, H. W. Robert, Geoff J. M. Parker, Daniel C. Alexander, Mark R. Symms, Philip A. Boulby, Claudia A. M. Wheeler-Kingshott, Gareth J. Barker, Uta Noppeney, Matthias J. Koepp, and John S. Duncan. 2006. 'Hemispheric Asymmetries in Language-Related Pathways: A Combined Functional MRI and Tractography Study'. NeuroImage 32(1):388-99. doi: 10.1016/j.neuroimage.2006.03.011. 
Pulvermüller, Friedemann. 2018. 'Neural Reuse of Action Perception Circuits for Language, Concepts and Communication'. Progress in Neurobiology 160:1-44. doi: 10.1016/j.pneurobio.2017.07.001.

Rilling, James, Matthew F. Glasser, Saad Jbabdi, Jesper Andersson, and Todd M. Preuss. 2012. 'Continuity, Divergence, and the Evolution of Brain Language Pathways'. Frontiers in Evolutionary Neuroscience 3. doi: 10.3389/fnevo.2011.00011.

Rilling, James K., Matthew F. Glasser, Todd M. Preuss, Xiangyang Ma, Tiejun Zhao, Xiaoping Hu, and Timothy E. J. Behrens. 2008. 'The Evolution of the Arcuate Fasciculus Revealed with Comparative DTI'. Nature Neuroscience 11(4):426-28. doi: 10.1038/nn2072.

Rocchi, Francesca, Hiroyuki Oya, Fabien Balezeau, Alexander J. Billig, Zsuzsanna Kocsis, Rick L. Jenison, Kirill V. Nourski, Christopher K. Kovach, Mitchell Steinschneider, Yukiko Kikuchi, Ariane E. Rhone, Brian J. Dlouhy, Hiroto Kawasaki, Ralph Adolphs, Jeremy D. W. Greenlee, Timothy D. Griffiths, Matthew A. Howard, and Christopher I. Petkov. 2021. 'Common FrontoTemporal Effective Connectivity in Humans and Monkeys'. Neuron. doi: 10.1016/j.neuron.2020.12.026.

Romanski, Lizabeth, Tian, Bailling, Fritz, Jonathan, Mishkin, Mortimer, Goldman-Rakic, Patricia, and Rauschecker, Joseph. 1999. 'Dual Streams of Auditory Afferents Target Multiple Domains in the Primate Prefrontal Cortex'. Nature Neuroscience 2(12):1131-36. doi: 10.1038/16056.

Roumazeilles, Lea, Nicole Eichert, Katherine L. Bryant, Davide Folloni, Jerome Sallet, Suhas Vijayakumar, Sean Foxley, Benjamin C. Tendler, Saad Jbabdi, Colin Reveley, Lennart Verhagen, Lori B. Dershowitz, Martin Guthrie, Edmund Flach, Karla L. Miller, and Rogier B. Mars. 2020. 'Longitudinal Connections and the Organization of the Temporal Cortex in Macaques, Great Apes, and Humans' edited by S. W. Townsend. PLOS Biology 18(7):e3000810. doi: 10.1371/journal.pbio.3000810.

Saleeba, Christine, Bowen Dempsey, Sheng Le, Ann Goodchild, and Simon McMullan. 2019. 'A Student's Guide to Neural Circuit Tracing'. Frontiers in Neuroscience 13:897. doi: 10.3389/fnins.2019.00897.

Saur, Dorothee, Björn W. Kreher, Susanne Schnell, Dorothee Kümmerer, Philipp Kellmeyer, MagnusSebastian Vry, Roza Umarova, Mariacristina Musso, Volkmar Glauche, Stefanie Abel, Walter Huber, Michel Rijntjes, Jürgen Hennig, and Cornelius Weiller. 2008. 'Ventral and Dorsal Pathways for Language'. Proceedings of the National Academy of Sciences 105(46):18035-40. doi: 10.1073/pnas.0805234105.

Schmahmann, Jeremy D., and Deepak N. Pandya. 2006. Fiber Pathways of the Brain. Oxford; New York: Oxford University Press.

Seltzer, Benjamin, and Pandya, Deepak. 1984. 'Further Observations on Parieto-Temporal Connections in the Rhesus Monkey’. Experimental Brain Research 55(2):301-12. doi: 10.1007/BF00237280.

Silva, Guilherme, and Alberto Citterio. 2017. 'Hemispheric Asymmetries in Dorsal Language Pathway White-Matter Tracts: A Magnetic Resonance Imaging Tractography and Functional Magnetic Resonance Imaging Study'. The Neuroradiology Journal 30(5):470-76. doi: $10.1177 / 1971400917720829$. 
Takaya, Shigetoshi, Gina R. Kuperberg, Hesheng Liu, Douglas N. Greve, Nikos Makris, and Steven M. Stufflebeam. 2015. 'Asymmetric Projections of the Arcuate Fasciculus to the Temporal Cortex Underlie Lateralized Language Function in the Human Brain'. Frontiers in Neuroanatomy 9:119. doi: 10.3389/fnana.2015.00119.

Tecumseh Fitch, W. 2018. 'Bio-Linguistics: Monkeys Break Through the Syntax Barrier'. Current Biology 28(12):R695-97. doi: 10.1016/j.cub.2018.04.087.

Thiebaut de Schotten, Michel, Flavio Dell'Acqua, Stephanie J. Forkel, Andrew Simmons, Francesco Vergani, Declan G. M. Murphy, and Marco Catani. 2011. 'A Lateralized Brain Network for Visuospatial Attention'. Nature Neuroscience 14(10):1245-46. doi: 10.1038/nn.2905.

Thiebaut de Schotten, Michel, Flavio Dell'Acqua, Romain Valabregue, and Marco Catani. 2012. 'Monkey to Human Comparative Anatomy of the Frontal Lobe Association Tracts'. Cortex 48(1):82-96. doi: 10.1016/j.cortex.2011.10.001.

Thiebaut de Schotten, Michel, Dominic H. ffytche, Alberto Bizzi, Flavio Dell'Acqua, Matthew Allin, Muriel Walshe, Robin Murray, Steven C. Williams, Declan G. M. Murphy, and Marco Catani. 2011. 'Atlasing Location, Asymmetry and Inter-Subject Variability of White Matter Tracts in the Human Brain with MR Diffusion Tractography'. NeuroImage 54(1):49-59. doi: 10.1016/j.neuroimage.2010.07.055.

Toga, Arthur W., and Paul M. Thompson. 2003. 'Mapping Brain Asymmetry'. Nature Reviews Neuroscience 4(1):37-48. doi: 10.1038/nrn1009.

Van Essen, David C., and Donna L. Dierker. 2007. 'Surface-Based and Probabilistic Atlases of Primate Cerebral Cortex'. Neuron 56(2):209-25. doi: 10.1016/j.neuron.2007.10.015.

Vavassori, Laura, Silvio Sarubbo, and Laurent Petit. 2021. 'Hodology of the Superior Longitudinal System of the Human Brain: A Historical Perspective, the Current Controversies, and a Proposal'. Brain Structure and Function. doi: 10.1007/s00429-021-02265-0.

Verhelst, Helena, Thijs Dhollander, Robin Gerrits, and Guy Vingerhoets. 2021. 'Fibre-Specific Laterality of White Matter in Left and Right Language Dominant People'. NeuroImage 117812. doi: 10.1016/j.neuroimage.2021.117812.

Vernooij, M. W., M. Smits, P. A. Wielopolski, G. C. Houston, G. P. Krestin, and A. van der Lugt. 2007. 'Fiber Density Asymmetry of the Arcuate Fasciculus in Relation to Functional Hemispheric Language Lateralization in Both Right- and Left-Handed Healthy Subjects: A Combined FMRI and DTI Study'. NeuroImage 35(3):1064-76. doi: 10.1016/j.neuroimage.2006.12.041.

von Economo, Constantin Freiherr, and Georg N. Koskinas. Die cytoarchitektonik der hirnrinde des erwachsenen menschen. J. Springer, 1925.

Wang, Yibao, Juan C. Fernández-Miranda, Timothy Verstynen, Sudhir Pathak, Walter Schneider, and Fang-Cheng Yeh. 2013. 'Rethinking the Role of the Middle Longitudinal Fascicle in Language and Auditory Pathways'. Cerebral Cortex 23(10):2347-56. doi: 10.1093/cercor/bhs225.

Warrington, Shaun, Katherine L. Bryant, Alexandr A. Khrapitchev, Jerome Sallet, Marina CharqueroBallester, Gwenaëlle Douaud, Saad Jbabdi, Rogier B. Mars, and Stamatios N. Sotiropoulos. 2020. 
'XTRACT - Standardised Protocols for Automated Tractography in the Human and Macaque Brain'. NeuroImage 217:116923. doi: 10.1016/j.neuroimage.2020.116923.

3 Wernicke, Carl. 1874. Der aphasische Symptomencomplex: Eine psychologische Studie auf anatomischer 4 Basis. Cohn.

5 Wilson, Benjamin, Yukiko Kikuchi, Li Sun, David Hunter, Frederic Dick, Kenny Smith, Alexander Thiele, Timothy D. Griffiths, William D. Marslen-Wilson, and Christopher I. Petkov. 2015. 'Auditory Sequence Processing Reveals Evolutionarily Conserved Regions of Frontal Cortex in Macaques and Humans'. Nature Communications 6(1):8901. doi: 10.1038/ncomms9901.

9 Yaşargil, M. Gazi, Ugur Türe, and Dianne C. H. Yaşargil. 2004. 'Impact of Temporal Lobe Surgery'. 10 Journal of Neurosurgery 101(5):725-38. doi: 10.3171/jns.2004.101.5.0725.

11 Zhu, Linlin, Yang Fan, Qihong Zou, Jue Wang, Jia-Hong Gao, and Zhendong Niu. 2014. 'Temporal 12 Reliability and Lateralization of the Resting-State Language Network'. PLOS ONE 9(1):e85880. doi: 10.1371/journal.pone.0085880. 